\title{
Modes of random lasers
}

\author{
J. Andreasen, ${ }^{1}$ A. A. Asatryan, ${ }^{2}$ L. C. Botten, ${ }^{2}$ M. A. Byrne, ${ }^{2}$ H. Cao, ${ }^{1}$ \\ L. Ge, ${ }^{1}$ L. Labonté, ${ }^{3}$ P. Sebbah, ${ }^{3, *}$ A. D. Stone, ${ }^{1}$ H. E. Türeci, ${ }^{4,5}$ and \\ C. Vanneste ${ }^{3}$ \\ ${ }^{1}$ Department of Applied Physics, P.O. Box 208284, Yale University, New Haven, \\ Connecticut 06520-8284, USA \\ ${ }^{2}$ Department of Mathematical Sciences and Center for Ultrahigh-bandwidth Devices for \\ Optical Systems (CUDOS), University of Technology, Sydney, \\ New South Wales 2007, Australia \\ ${ }^{3}$ Laboratoire de Physique de la Matière Condensée, CNRS UMR 6622, Université de \\ Nice-Sophia Antipolis, Parc Valrose, 06108 Nice Cedex 02, France \\ ${ }^{4}$ Institute for Quantum Electronics, ETH Zurich, 8093 Zurich, Switzerland \\ ${ }^{5}$ Department of Electrical Engineering, Princeton University, Princeton, New Jersey \\ 08544, USA
}

*Corresponding author: sebbah@unice.fr

Received January 21, 2010; revised August 4, 2010; accepted August 6, 2010;

published October 1, 2010 (Doc. ID 123020)

In conventional lasers, the optical cavity that confines the photons also determines essential characteristics of the lasing modes such as wavelength, emission pattern, directivity, and polarization. In random lasers, which do not have mirrors or a well-defined cavity, light is confined within the gain medium by means of multiple scattering. The sharp peaks in the emission spectra of semiconductor powders, first observed in 1999, has therefore lead to an intense debate about the nature of the lasing modes in these so-called lasers with resonant feedback. We review numerical and theoretical studies aimed at clarifying the nature of the lasing modes in disordered scattering systems with gain. The past decade has witnessed the emergence of the idea that even the low- $Q$ resonances of such open systems could play a role similar to the cavity modes of a conventional laser and produce sharp lasing peaks. We focus here on the nearthreshold single-mode lasing regime where nonlinear effects associated with gain saturation and mode competition can be neglected. We discuss in particular the link between random laser modes near threshold and the resonances or quasi-bound (QB) states of the passive system without gain. For random lasers in the localized (strong scattering) regime, QB states and threshold lasing modes were found to be nearly identical within the scattering medium. These studies were later extended to the case of more lossy systems such as random systems in the diffusive regime, where it was observed that increasing the openness of such systems eventually resulted in measurable and increasing differences between quasi-bound states and lasing modes. Very recently, a theory able to treat lasers with arbitrarily complex and open cavities such as random lasers established that the threshold lasing modes are in fact distinct from QB states of the passive system and are better described in terms of a new class of 
states, the so-called constant-flux states. The correspondence between QB states and lasing modes is found to improve in the strong scattering limit, confirming the validity of initial work in the strong scattering limit. (C) 2011 Optical Society of America

OCIS codes: $140.3460,140.3430,290.4210,260.2710$.

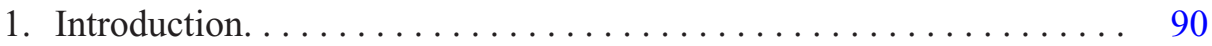

2. Early Numerical Explorations: Time-Dependent Model. . . . . . . . . . 92

2.1. Localized Case . . . . . . . . . . . . . . . . . . . . . . . 92

2.2. Diffusive Case. ........................... 95

3. Numerical Simulations: Time-Independent Models. . . . . . . . . . . . 99

3.1. One-Dimensional Random Lasers. . . . . . . . . . . . . . . . . . . . . . 99

3.2. Two-Dimensional Random Lasers. . . . . . . . . . . . . . . . 102

3.2a. Localized Case. . . . . . . . . . . . . . . . . . . . . . 102

3.2b. Diffusive Case. . . . . . . . . . . . . . . . . . . . . . . . . . 103

3.2c. Transition Case. . . . . . . . . . . . . . . . . . . . 104

4. Threshold Lasing States versus Passive Cavity Resonances. ... . . . . . 107

5. Self-Consistent Time-Independent Approach to Random Lasing. . . . . 109

5.1. Maxwell-Bloch Threshold Lasing Modes. . . . . . . . . . . . . . 109

5.2. Self-Consistent Steady-State Lasing Equations. ........... 110

5.3. Solution for Threshold Lasing Modes and Constant-Flux States... 112

5.4. Nonlinear Steady-State ab Initio Laser Theory. . . . . . . . . . . 114

6. Conclusion. ............................... 117

Appendix A: Multipole Method. . . . . . . . . . . . . . . . . . . . . . 117

Appendix B: Finite Element Method. . . . . . . . . . . . . . . . . . 120

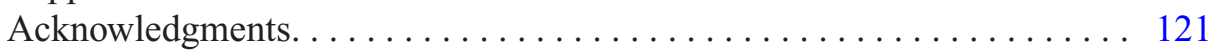

References........................... 121 


\title{
Modes of random lasers
}

\author{
J. Andreasen, A. A. Asatryan, L. C. Botten, M. A. Byrne, H. Cao, L. Ge, \\ L. Labonté, P. Sebbah, A. D. Stone, H. E. Türeci, and C. Vanneste
}

\section{Introduction}

The investigation of laser action in complex media with disorder has a long history going back to the early days of laser physics (for a review, see [1-5]). Beginning in the mid 1990s there was a resurgence of interest in this topic both for its intrinsic interest and because of a possible relation to the phenomenon of Anderson localization [6], previously studied mainly in the context of electronic systems. Random lasers are disordered media with gain that do not possess a light-trapping cavity beyond the confinement provided by multiple-scattering from the disorder itself. Hence they are usually extremely open, low-finesse lasers. Initially it was unclear whether such systems could produce narrow lasing lines without any well-confined electromagnetic modes, and while initial experimental studies did find strong amplification near the transition frequency determined by the gain medium, discrete lines were not observed [7-9]. Subsequent studies in smaller systems with focused pumping did find discrete lasing lines, not necessarily located at the center of the gain curve, and photon statistics characteristic of gain saturation [10-15], demonstrating that in some cases random lasers behave very much like conventional multimode lasers except for their relatively high thresholds and their pseudorandom emission patterns in space. The experimental observations of laser peaks have naturally called for the search for a feedback mechanism leading to light trapping within the scattering medium. There is in fact a case where light can be well confined inside an open disordered medium. Such confinement occurs when the scattering is extremely strong and the system is in the regime of Anderson localization [16]. However, except in quasi-1D geometries [17], the vast majority of experiments on random lasers do not appear to be in the localized regime, so the question of whether laser action in a diffusive $(L \gg \ell)$ or quasi-ballistic $(L \sim \ell)$ medium has a qualitatively different nature and origin with respect to conventional lasers remained open for some time (here $L$ is the system size and $\ell$ is the optical elastic mean free path).

With the renewed experimental interest in random lasers came also a number of attempts to generalize laser theory to describe such a system. Early on a major distinction was made between conventional lasers, which operate on resonant feedback, and random lasers, which at least in some cases were supposed to operate only on nonresonant feedback [4]. In the case of nonresonant feedback the light intensity in the laser was described by a diffusion equation with gain, but the phase of the light field and hence interference did not play a role. A key finding is that there is a threshold for amplification when the diffusion length for es- 
cape $L_{D} \sim d L^{2} / \ell$ becomes longer than the gain length (here $d=2,3$ is the dimensionality). The spatial distribution of intensity above threshold would be given by the solution of a diffusion equation. In this approach there would be no frequency selectivity, and the amplified light would peak at the gain center. Clearly such a description would be inadequate to describe random lasers based on Anderson localized modes, as such modes are localized in space precisely owing to destructive interference of diffusing waves arising from multiple scattering.

This question itself is related to a basic question in nonlinear optics: can a system, disordered or not, which is so leaky that it has no isolated linear scattering resonances, nonetheless have sharp laser lines due to the addition of gain? And if so, how are the modes associated with these lines related to the broad and difficult to observe resonances of the passive cavity? For an open diffusive or quasiballistic medium in two or three dimensions the resonance spacing in the wave vector will decrease as $\lambda^{d-1} / L^{d}$, whereas the linewidth will scale as $\ell / L^{2}$ (diffusive) or as $1 / L$ (ballistic). Therefore (unless $\ell \approx \lambda$ in $d=2$ ) the disordered passive cavity resonances strongly overlap and cannot be directly observed in linear scattering.

In the search for a feedback mechanism responsible for the sharp laser peaks observed experimentally [18], different scenarios have been proposed. As an alternative to the early picture of closed scattering loops, the probability of having ring-shaped resonators with index of refraction larger than average in the diffusive regime was calculated and shown to be substantially increased by disorder correlation due to finite-size scatterers $[19,20]$. Another scenario was put forward where spontaneously emitted photons accumulate gain along very long trajectories. This follows the observation of random spikes in the emission spectrum of weakly active scattering systems in single-shot experiments $[21,22]$. These "lucky photons" accumulate enough gain to activate a new lasing mode with a different wavelength after each excitation shot. The experimental study of the modal decay rates in microwave experiments leading to the observation of anomalous diffusion has brought forward the existence of longer-lived prelocalized modes in an otherwise diffusive system [23]. An experimental indication of the coexistence of extended and localized lasing modes was presented recently [24]. It was suggested that these longer-lived modes could be responsible for lasing. However, although they are possibly achieved in some specific situations, those different scenarios cannot explain the whole set of experimental observations

In this paper, we present recent work, both numerical and analytical, which has shown that within semiclassical laser theory, in which the effects of quantum noise are neglected, definite answers to these questions can be given, without resorting to exotic scenarios. Sharp laser lines based on interference (coherent feedback) do exist, not only in strongly scattering random lasers where the localized regime is reached [25-27], but also in diffusive random lasers [28,29] and even for weak scattering [30]. Numerical studies have shown that they are associated with threshold lasing modes (TLMs), which, inside the cavity, are similar to the resonances or quasi-bound (QB) states of the passive system (also called quasi-normal modes). The resemblance is excellent in the localized case $[26,27]$ and deteriorates as scattering is reduced. A new theoretical approach based on a reformulation of the Maxwell-Bloch (MB) equations to access the steady-state properties of arbitrarily complex and open cavities allows one to calculate the lasing modes in diffusive and even in weakly scattering random lasers $(\ell \sim L)[31-35]$. A major outcome of this approach is the demonstration that 
although lasing modes and passive modes can be very alike in random systems with moderate openness, in agreement with the above numerical results, they feature fundamental differences. Their distinctness increases with the openness of the random system and becomes substantial for weakly scattering systems. Constant-flux (CF) states are introduced that better describe TLMs both inside and outside the scattering medium for any scattering strength. In addition this theoretical approach allows one to study the multimode regime in diffusive random lasers and get detailed information about the effects of mode competition through spatial hole burning, which appear to differ from those for conventional lasers.

In this past decade, different types of random lasers (semiconductor powders, piconjugated polymers, scattering suspension in dyes, random microcavities, dyedoped nematic liquid crystals, random fiber lasers...) have been considered in the literature. We will focus throughout this review mostly on 2D random lasers that consist of randomly distributed dielectric nanoparticles as scatterers. This choice makes possible the numerical and theoretical exploration of $2 \mathrm{D}$ finitesized opened samples where transport can be made ballistic, diffusive (in contrast to 1D), or localized [36] by adjusting the index contrast between the scatterers and the background medium.

The outline of this review is as follows: in Section 2 we review early numerical explorations of localized and diffusive random lasing demonstrating the existence of TLMs in all regimes. In Section 3 we present recent numerical work based on a time-independent model, which indicates the difference between passive cavity resonances and TLMs, discussing only single-mode random lasing. The following section will explain why, in principle, QB states cannot describe TLMs. Section 5 will introduce the concept of CF states and describe the selfconsistent time-independent approach to describe random lasing modes at threshold as well as in the multimode regime.

\section{Early Numerical Explorations: Time-Dependent Model}

\subsection{Localized Case}

From a modal point of view, Anderson localization means that for strong disorder, the eigenmodes of the wave equation are spatially localized in a volume of finite size $2 \xi$, where $\xi$ is the localization length. More precisely, they are spatially localized solutions of the Maxwell equations with tails, which decay exponentially from their center, $\xi$ being the decay length. In the case of scattering particles, the value of the localization length is controlled by the index contrast between the particles and the background medium, the size of the particles, the optical wavelength, and the amount of disorder. In practice, when finite-size systems in the localization regime are considered, two opposite cases may occur: (1) $\xi>L$ and (2) $\xi<L$, where $L$ is the system size. In the first case, the system is not large enough for the light to be confined by disorder within the volume of the system. In case (2), light is localized, since it cannot escape domains larger than $\xi$. More precisely, localized modes are coupled to the boundaries via their exponential tails. The leakage rate of an exponentially localized QB state varies as $\exp (-2 r / \xi)$ with $r$ the distance to the boundaries [37]. Hence, in sufficiently large systems QB states located far from the boundaries (which constitutes majority of the 
QB states except a fraction proportional to $\xi / L$ ) feature a very small leakage, i.e., a good quality factor.

In this subsection, we will consider case (2). Localized modes in a disordered scattering system are quite like the modes of standard optical cavities, such as the Fabry-Perot [38]. Hence, one can expect that in the presence of gain the lasing modes in this regime of strong disorder will be close to the localized QB states of the passive system without gain, in the same way as the lasing modes of a conventional cavity are built with the QB states of the passive cavity. To verify that this is really the case one must have access to the individual modes of both the passive system and the active system. Experimentally, such a demonstration has not been achieved yet, essentially because the regime of Anderson localization is difficult to reach and to observe in optics. Besides, until recently there was no fully developed theory describing random lasing modes and their relationship with the eigenstates of the passive system. The easiest way to check this conjecture has been to resort to numerical simulations.

Historically, most of the early numerical studies of random lasers were based on the diffusion equation (see references in [4]). However, it is not possible to take into account under the diffusion approximation the interference phenomena that are at the heart of Anderson localization. This is why Jiang and Soukoulis [25] proposed to solve the time-dependent Maxwell equations coupled with the population equations of a four-level system [40]. The populations $N_{i}$, $i=1$ to $i=4$ satisfy the following equations:

$$
\begin{aligned}
& d N_{1} / d t=N_{2} / \tau_{21}-W_{p} N_{1}, \\
& d N_{2} / d t=N_{3} / \tau_{32}-N_{2} / \tau_{21}-\left(\mathbf{E} / \hbar \omega_{a}\right) d \mathbf{P} / d t, \\
& d N_{3} / d t=N_{4} / \tau_{43}-N_{3} / \tau_{32}+\left(\mathbf{E} / \hbar \omega_{a}\right) d \mathbf{P} / d t, \\
& d N_{4} / d t=-N_{4} / \tau_{43}+W_{p} N_{1},
\end{aligned}
$$

where $W_{p}$ is the rate of an external mechanism that pumps electrons from the fundamental level (1) to the upper level (4). The electrons in level 4 relax quickly with time constant $\tau_{43}$ to level 3 . The laser transition occurs from level 3 to level 2 at frequency $\omega_{a}$. Hence, electrons in level 3 can jump to level 2 either spontaneously with time constant $\tau_{32}$ or through stimulated emission with the rate $\left(\mathbf{E} / \hbar \omega_{a}\right) d \mathbf{P} / d t$. $\mathbf{E}$ and $\mathbf{P}$ are the electric field and the polarization density, respectively. Eventually, electrons in level 2 relax quickly with time constant $\tau_{21}$ from level 2 to level 1 . In these equations, the populations $N_{i}$, the electric field $\mathbf{E}$, and the polarization density $\mathbf{P}$ are functions of the position $\mathbf{r}$ and the time $t$.

The polarization obeys the equation

$$
d^{2} \mathbf{P} / d t^{2}+\Delta \omega_{a} d \mathbf{P} / d t+\omega_{a}^{2} \mathbf{P}=\kappa \cdot \Delta N \cdot \mathbf{E},
$$

where $\Delta N=N_{2}-N_{3}$ is the population density difference. Amplification takes place when the rate $W_{p}$ of the external pumping mechanism produces inverted population difference $\Delta N<0$. The linewidth of the atomic transition is $\Delta \omega_{a}$ $=1 / \tau_{32}+2 / T_{2}$, where the collision time $T_{2}$ is usually much smaller than the lifetime $\tau_{32}$. The constant $\kappa$ is given by $\kappa=3 c^{3} / 2 \omega_{a}^{2} \tau_{32}$ [40].

Finally, the polarization is a source term in the Maxwell equations, 


$$
\begin{gathered}
\partial \mathbf{H} / \partial t=-c \nabla \times \mathbf{E} \\
\epsilon(\mathbf{r}) \partial \mathbf{E} / \partial t=c \nabla \times \mathbf{H}-4 \pi \partial \mathbf{P} / \partial t .
\end{gathered}
$$

The randomness of the system arises from the dielectric constant $\epsilon(\mathbf{r})$, which depends on the position $\mathbf{r}$. This time-dependent model has been used in random 1D systems consisting of a random stack of dielectric layers separated by gain media [25] and in random 2D systems consisting of a random collection of circular particles embedded in a gain medium (Fig. 1) [26]. In both cases, a large optical index contrast has been assigned between the scatterers and the background medium to make sure that the regime of Anderson localization was reached. The Maxwell equations are solved by using the finite-difference time-domain method (FDTD) [41]. To simulate an open system, perfectly matched layers are introduced at the boundaries of the system [42]. The pumping rate $W_{p}$ is adjusted just above lasing threshold in order to remain in the single-mode regime.

In one dimension, the QB states of the passive system were obtained independently using a time-independent transfer matrix method [43]. In two dimensions, the Maxwell equations were solved without the polarization term in Eq. (7), again using the FDTD method. First, the spectrum of eigenfrequencies was obtained by Fourier transform of the impulse response of the system. Next, QB states were excited individually by a monochromatic source at each of the eigenfrequencies.

Finally, in 1D systems [43] as well as in 2D systems [27], lasing modes obtained by the full time-dependent model with gain and localized QB states of the corresponding passive system without gain were compared and found to be identical with a good precision. This was verified for all modes obtained by changing the disorder configuration. An example of a 2D lasing mode and the corresponding QB state of the same system (Fig. 1) without gain are displayed in Fig. 2.

\section{Figure 1}

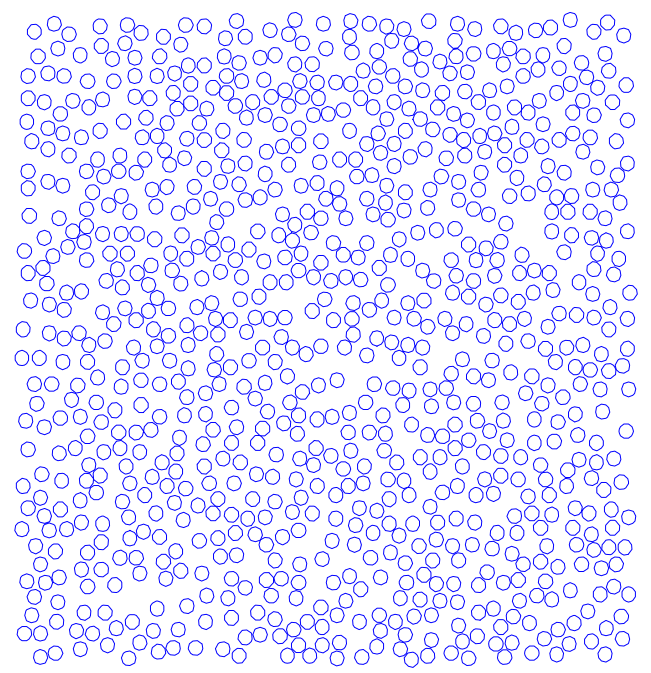

Example of a random realization of 896 circular scatterers contained in a square box of size $L=5 \mu \mathrm{m}$ and optical index $n=1$. The radius and the optical index of the scatterers are, respectively, $r=60 \mathrm{~nm}$ and $n=2$. The total system of size $9 \mu \mathrm{m}$ is bounded by perfectly matched layers (not shown) to simulate an open system. 


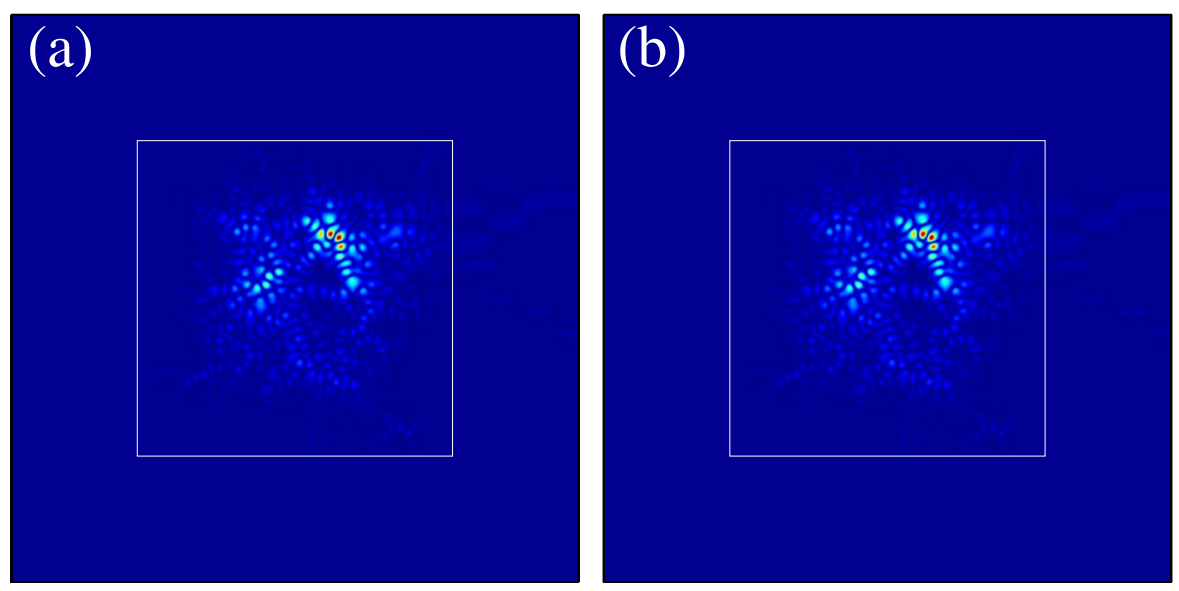

(a) Spatial distribution of the amplitude of a lasing mode in the localized regime $(n=2)$ and (b) that of the corresponding QB states of the same random system without gain. The squares delimit the scattering medium. The amplitude rather than the intensity is represented for a better display of the small values of the field.

These results confirmed that the QB states of a localized system play a role similar to the eigenmodes of the cavity of a conventional laser. The only difference is the complicated and system-dependent nature of the localized modes as opposed to the well-known modes of a conventional cavity. These results are in good agreement with the theoretical results described in Section 5, which show that inside systems in the localized regime, the single lasing modes just above threshold are close to the high- $Q$ resonances of the passive system.

\subsection{Diffusive Case}

We have seen in the previous section that random lasers in the Anderson localization regime should behave like conventional lasers. They should exhibit discrete laser peaks above threshold in agreement with the experimental observations of laser action with resonant feedback. However, subsequent measurements of the mean free path showed that none of the experimental cases that displayed discrete laser peaks were in the localized regime. Instead, they were found to be in the diffusive regime and some even in the quasi-ballistic regime [30]. In such systems, there are no localized modes, so that the observation of laser action with resonant feedback has been the subject of much debate.

Only very recently, numerical evidence was given that even diffusive systems with low- $Q$ resonances could exhibit lasing with resonant feedback [28]. The random $2 \mathrm{D}$ systems described in the previous subsection consisting of random collections of circular particles embedded in a gain medium have been investigated with the same time-dependent model. To be in the diffusive regime instead of the localized regime, a smaller optical index contrast $\delta_{n}=0.25$ instead of $\delta_{n}$ $=1.0$ has been assigned between the scatterers and the background medium. Solving the Maxwell equations coupled to the population equations, laser action characterized by a sharp peak in the emission spectrum was observed just above a threshold, albeit high. An example of the corresponding lasing mode is displayed in Fig. 3(a). 

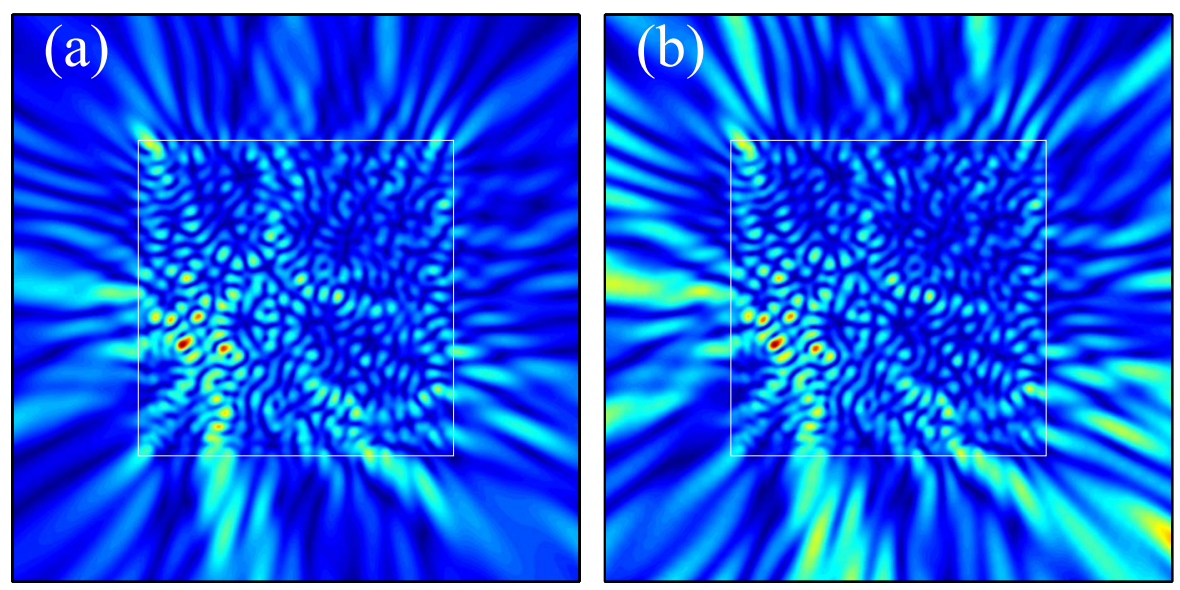

(a) Spatial distribution of the amplitude of a lasing mode in the diffusive regime.

(b) Spatial distribution of the field amplitude after the pump has been stopped and the polarization term has been set to zero. The spatial distribution of scatterers is the collection shown in Fig. 1, but here the optical index of the scatterers is $n=1.25$ instead of $n=2$ in Fig. 2

In contrast to the localized case, the lasing mode is now extended over the whole system. Moreover, this is a complex mode in the sense that it contains a substantial traveling wave component [28]. However, in this work comparison of the lasing modes with the QB states of the passive cavity could not be carried out by using the time domain method as it was done in the localized regime. Due to strong leakage through the boundaries, resonances are strongly overlapping in the frequency domain, and one cannot excite them individually by a monochromatic source.

To circumvent this difficulty, an indirect method has been used to compare the lasing modes with the resonances of the passive system. This method is inspired by the Fox-Li modes, which in conventional laser physics are modes of an open cavity [40,44-46]. The Fox-Li modes are field distributions whose profile is selfrepeating in a complete round trip within the Fabry-Perot laser cavity while decaying because of the diffraction losses due to finite surface area of the end mirrors. Analogously, if the lasing modes of the diffuse system are related to the resonances of the passive system, they should decay by self-repeating themselves when pumping and population inversion are turned off. To study the evolution of the mode profile with time, the following spatial correlation function was introduced [28]:

$$
C_{\mathcal{E}}\left(t_{0}, t\right)=\iint_{\mathcal{D}} d^{2} \vec{r} \mathcal{E}\left(\vec{r}, t_{0}\right) \mathcal{E}(\vec{r}, t)
$$

which compares the mode profile $\mathcal{E}(\vec{r}, t)$ at time $t$ with the mode profile at the initial time $t_{0}$. Here, $\mathcal{D}$ is the scattering medium. The field has been normalized, $\mathcal{E}(\vec{r}, t)=E(\vec{r}, t) /\left[\iint_{\mathcal{D}} d^{2} \vec{r} E^{2}(\vec{r}, t)\right]^{1 / 2}$, to counterbalance the decay due to the leakage through the boundaries. This correlation function oscillates at the laser frequency between -1 and +1 if the normalized mode profile is recovered at each period (Fig. 4). Otherwise, the amplitude of the oscillations should decay with time. This correlation function was used in [28] to check whether the first lasing mode at threshold for diffusive random laser indeed corresponds to a Fox-Li 


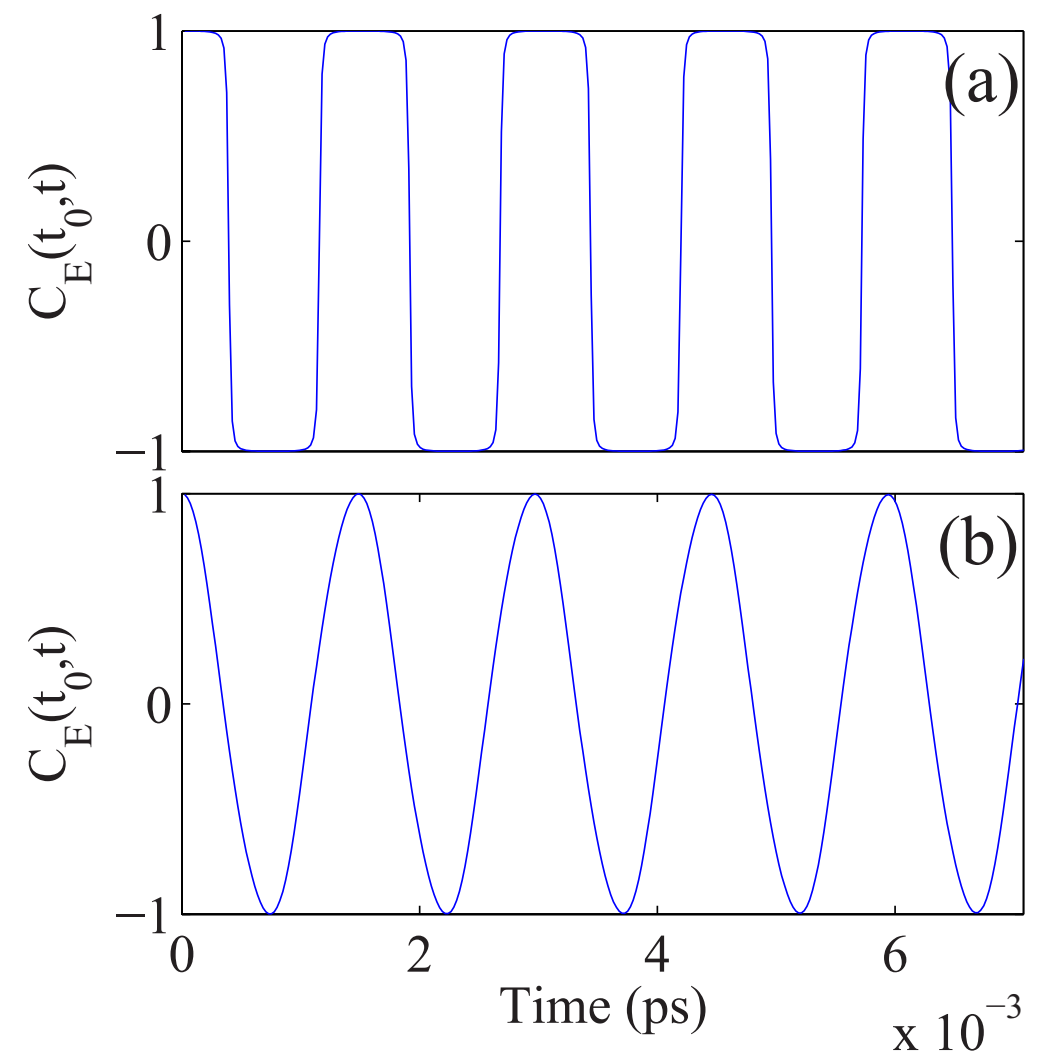

Short-time behavior over a few cycles of the correlation function, $C_{\mathcal{E}}\left(t_{0}, t\right)$, for (a) a localized lasing mode as in Fig. 2 and for (b) a diffusive lasing mode as in Fig. 3. The periodic square function in (a) is typical of a standing wave, while the sine-like function in (b) is characteristic of a traveling wave [28].

mode of the passive system. The pumping is set to zero after the lasing mode has been established so that at later times the field can evolve by itself. The long time evolution of the spatial correlation function associated with this free field is displayed in Fig. 5(a). The decay of the total energy of the system is also shown. While energy decay is observed over 6 orders of magnitude, the spatial correlation function is seen to oscillate between values close to -1 and +1 , meaning that the initial lasing mode profile $\mathcal{E}\left(\vec{r}, t_{0}\right)$ is reproduced at each period with a good accuracy. The decaying field amplitude has the spatial distribution that is shown in Fig. 3(b) until, eventually, the correlation function decays to zero when the decaying field reaches the noise level. This result demonstrates that the TLM is very close to a resonance of the passive system when measured inside the scattering medium. For comparison, the evolution of the spatial correlation function for an initial field created by an arbitrary distribution of monochromatic sources at the laser frequency is displayed in Fig. 5(b). The fast decay of $C_{\mathcal{E}}\left(t_{0}, t\right)$ after the sources have been turned off indicates that this field distribution is not a QB state of the passive system.

The decay rate observed corresponds to a quality factor of 30 , to be compared with the value $10^{4}$ found in the localized case. This result shows that a bad resonance in a leaky disordered system can nevertheless turn into a lasing mode in the presence of an active medium. This result is in stark contrast with the common belief 


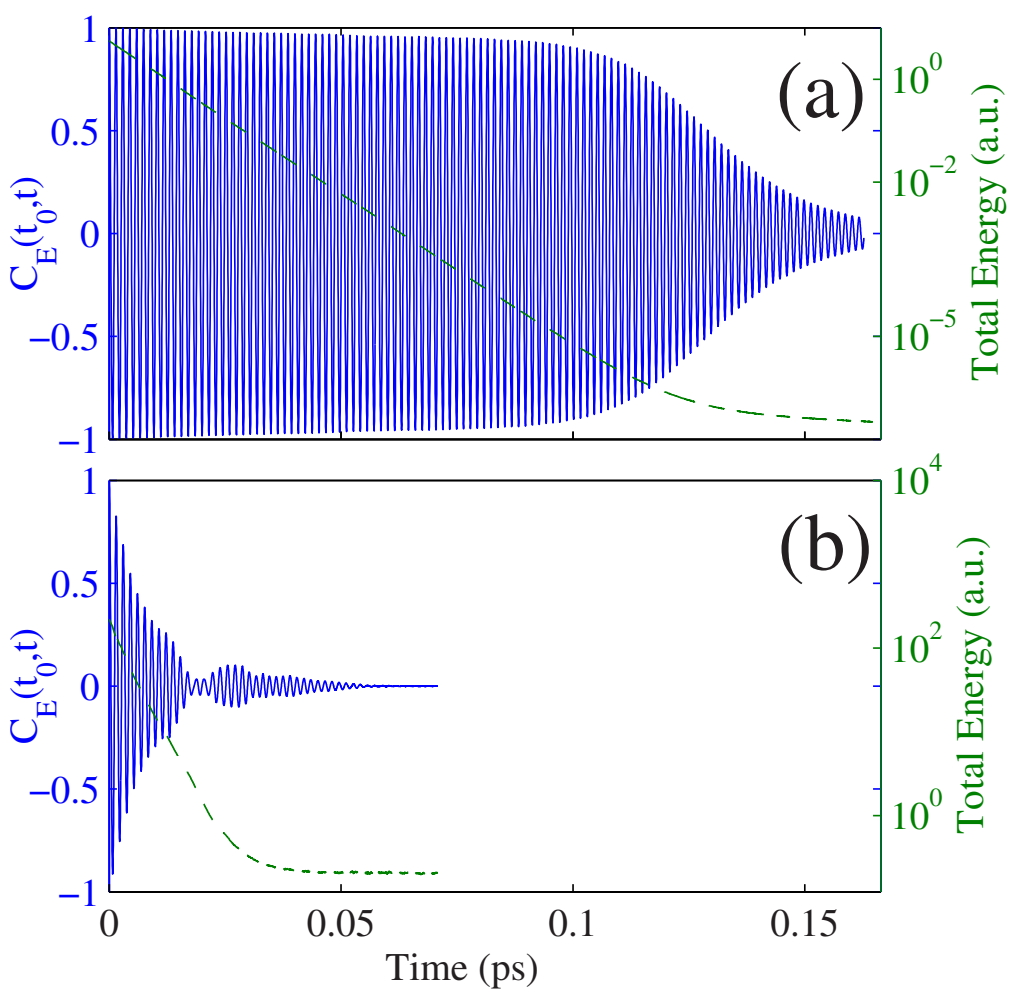

Correlation function (solid curves) and energy decay (dashed curves) versus time of (a) the lasing mode when the pump is turned off and (b) an arbitrary field distribution at the frequency of the lasing mode.

that random lasing with resonant feedback involves the presence of resonances with high quality factors. It provides a consistent explanation for the experimental observation of random lasing with resonant feedback even far from the localized regime, without resorting to other scenarios such as those reviewed in Section 1 [19-22].

The comparison of patterns between Fig. 3(a) and Fig. 3(b) shows that the lasing mode and the QB modes are close to each other inside the scattering system as confirmed by the evolution of the correlation function, which has been defined only inside the system. However, one also notices that outside the scattering medium the field distributions differ substantially. The free propagating field outside the scattering system in Fig. 3(b) reproduces the laser field distribution in Fig. 3(a) with significant distortions that are due to the enhancement of the amplitude towards the external boundaries of the total system. Hence, the comparison between both figures indicates that if the lasing modes and the QB modes are similar inside the scattering system, they differ noticeably outside. Moreover, a careful examination of the correlation function in Fig. 5(a) shows that it oscillates between two extremal values, which slowly depart from -1 and +1 well before the ultimate fast decay. This is in contrast with the long time behavior of the correlation function in the localized regime (not shown), which displays oscillations between -1 and +1 with a very good precision for time scales much longer than the time scale in Fig. 5(a). This result also indicates that inside the scattering system, the lasing mode is close to but not identical to a QB state. 
In conclusion, the time-dependent model has provided direct evidence of the closeness of lasing modes and passive cavity resonances, at least in the localized case. In the diffusive regime, the lasing modes are also found rather close to the QB modes, although small discrepancies manifest themselves. We also found that this holds inside the scattering medium. Outside the scattering system, however, differences become more significant. The advantage of the time-dependent model is that one has access in principle to the full nonlinear dynamics of the laser system. However, QB states with low quality factors are not accessible with this approach. Hence, the measure of the difference between TLM and QB states has been indirectly achieved by using the spatial correlation function. Another limitation of this method is related to the various time constants involved in this model, which lead to time-consuming computations, particularly when one wishes to vary disorder and study an ensemble of disorder configurations. To overcome these limitations, different approaches such as solving the wave equation in the frequency domain have been used. Several approaches of this kind will be described in the next section [14,47-49]. The recent theoretical approach based on a different class of states, the so-called constant flux (CF) states, and taking into account nonlinear interactions, will be described in Section 5.

\section{Numerical Simulations: Time-Independent Models}

Different models have been proposed in the frequency domain to solve the wave equation. In one dimension, it is possible to employ the transfer matrix method similar to that used in [43] for studying the lasing modes in an active layered random system. A direct comparison between TLMs and QB states of the corresponding passive random system is proposed in the first part of this section. In two dimensions, the multipole method has been used, which also provides a direct comparison of the QB states and the lasing modes of a 2D disordered open system. The comparison presented in the second part of this section has been carried out for refractive index of the scatterers ranging from $n_{l}^{\prime}=2.0$ (localized regime) to $n_{l}^{\prime}=1.25$ (diffusive regime). We alternatively used a different approach based on the finite element method to obtain the passive modes, which turned out to be much less computationally demanding in the weakly scattering regime. A brief description of both methods is provided in Appendices A and B.

\subsection{One-Dimensional Random Lasers}

Employing the transfer matrix method, similar to that used in [43], we study the lasing modes in a 1D random system and compare them with the QB states of the passive random system. The random system is composed of 161 layers. A dielectric material with index of refraction $n_{1}=1.05$ separated by air gaps $\left(n_{2}=1\right)$ results in a spatially modulated index of refraction $n(x)$. Outside the random medium $n_{0}=1$. The system is randomized by specifying thicknesses for each layer as $d_{1,2}=\left\langle d_{1,2}\right\rangle(1+\eta \zeta)$, where $\left\langle d_{1}\right\rangle=100 \mathrm{~nm}$ and $\left\langle d_{2}\right\rangle=200 \mathrm{~nm}$ are the average thicknesses of the layers, $\eta=0.9$ represents the degree of randomness, and $\zeta$ is a random number in $(-1,1)$. The length of the random structure $L$ is normalized to $\langle L\rangle$ $=24,100 \mathrm{~nm}$. Linear gain is simulated by appending an imaginary part to the dielectric function $\epsilon(x)=\epsilon^{\prime}(x)+i \epsilon^{\prime \prime}(x)$, where $\epsilon^{\prime}(x)=n^{2}(x)$. This approximation is valid at 
or below threshold [49]. The complex index of refraction is given by $\tilde{n}(x)=\sqrt{\epsilon(x)}$ $=n^{\prime}(x)+i n^{\prime \prime}$, where $n^{\prime \prime}<0$. We consider $n^{\prime \prime}$ to be constant everywhere within the random system. This yields a gain length $l_{g}=\left|1 / k^{\prime \prime}\right|=1 /\left|n^{\prime \prime}\right| k(k=2 \pi / \lambda$ is the vacuum frequency of a lasing mode), which is the same in the dielectric layers and the air gaps. The real part of the index of refraction is modified by the imaginary part as $n^{\prime}(x)=\sqrt{n^{2}(x)+n^{\prime \prime}}$.

We find the frequency $k$ and threshold gain $k^{\prime \prime}$ of each lasing mode within the wavelength range $500 \mathrm{~nm}<\lambda<750 \mathrm{~nm}$. The results are shown in Fig. 6. Finding matching QB states for lasing modes with large thresholds (large $\left|k^{\prime \prime}\right|$ ) is challenging because of large shifts of the solution locations [Fig. 6, region (c)]. However, there is a clear one-to-one correspondence with QB states for the lasing modes remaining [Fig. 6, regions (a) and (b)]. It is straightforward to find the matching QB states for these lasing modes and calculate their differences. The average percent difference between QB state frequencies and lasing mode frequencies in Fig. 6, region (a), is $0.013 \%$, while it is $0.15 \%$ in Fig. 6, region (b). The average percent difference between QB state decay rates $k_{0}^{\prime \prime}$ and lasing thresholds $k^{\prime \prime}$ in Fig. 6, region (a), is 2.5\% and in Fig. 6, region (b), is $21 \%$.

The normalized intensities of the QB states $I_{\mathrm{QB}}$ and lasing modes with linear gain $I_{\mathrm{LG}}$ are also compared. Figure 7 shows representative pairs of modes from the three regions shown in Fig. 6. The spatially averaged relative difference between each pair of modes is calculated by

\section{Figure 6}

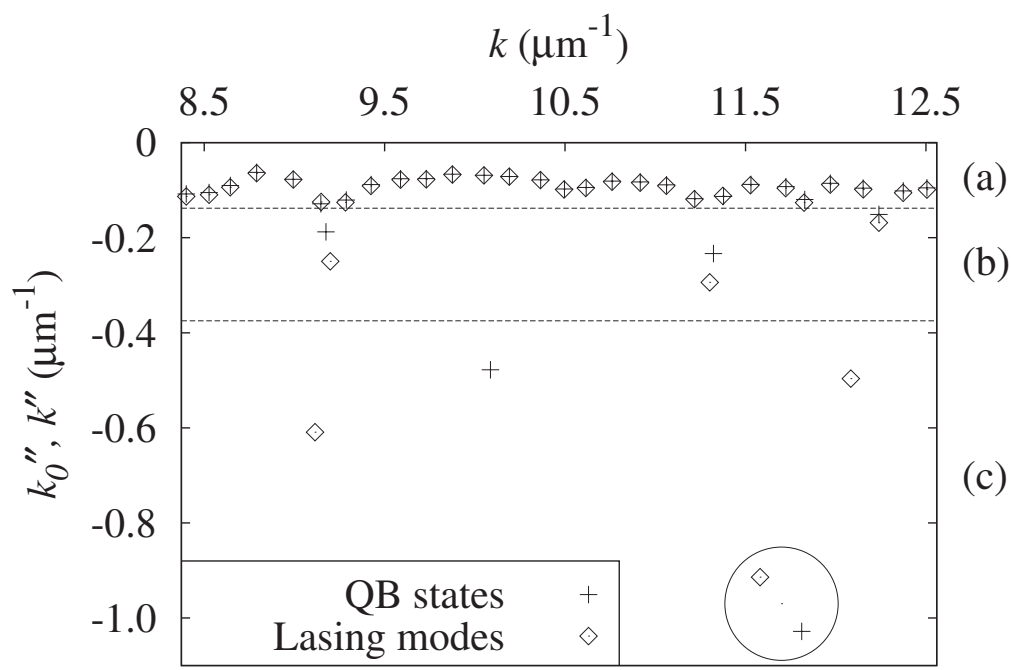

The frequencies $k$ of QB states (crosses) and lasing modes with linear gain (open diamonds) together with the decay rates $k_{0}^{\prime \prime}$ of QB states and the lasing thresholds $k^{\prime \prime}$ of lasing modes. The horizontal dashed lines separate three different regions of behavior: (a) lasing modes are easily matched to QB states, (b) clear differences appear but matching lasing modes to QB states is still possible, (c) lasing modes have shifted so much it is difficult to match them to QB states. The QB state with the largest decay rate and the lasing mode with the largest threshold are circled, though they may not be a matching pair. 


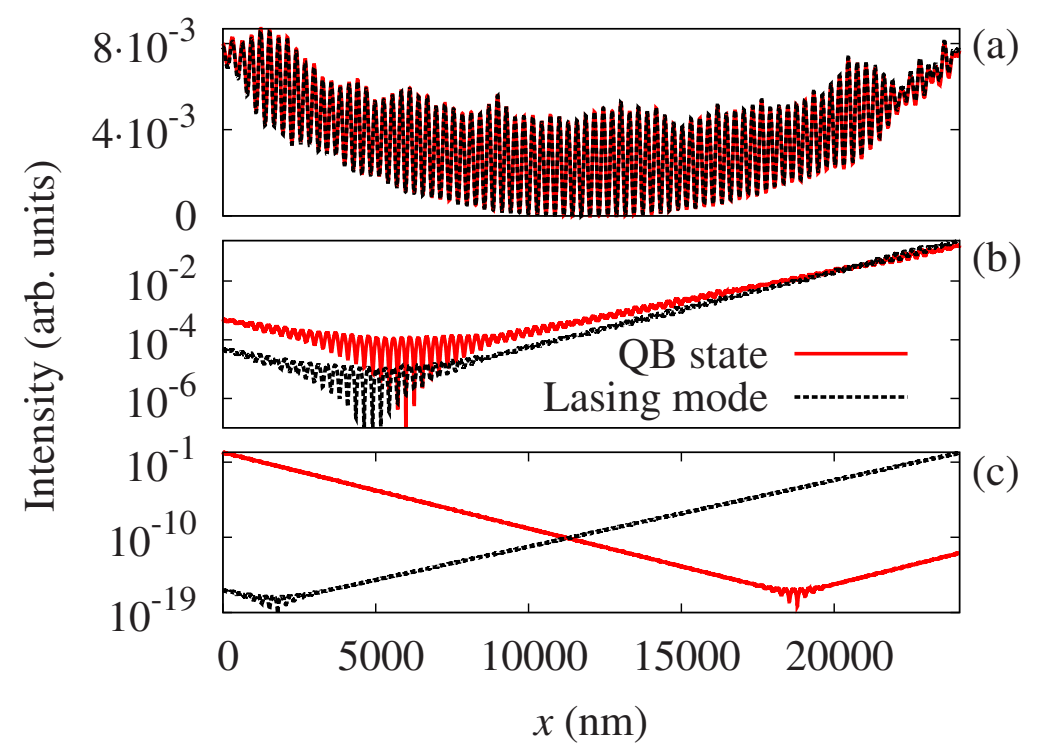

Spatial intensity distributions of $\mathrm{QB}$ states $I_{\mathrm{QB}}(x)$ (red solid lines) and lasing modes $I_{\mathrm{LG}}(x)$ (black dashed lines) from each of the three regions in Fig. 6. Representative samples were chosen for each case. (a) The lasing mode intensity is nearly identical to the QB state intensity with $\sigma_{d}=1.7 \%$. (b) A clear difference appears between the lasing mode and the QB state, with $\sigma_{d}=21.8 \%$, but they are still similar. (c) The lasing mode with the largest threshold and QB state with the largest decay rate are compared, with $\sigma_{d}=198 \%$. Though these two modes are fairly close to each other [circled in Fig. 6 region (c)], their intensity distributions are quite different.

$$
\left\langle\sigma_{d}\right\rangle=\frac{\int\left|I_{Q B}-I_{L G}\right| d A}{\int I_{L G} d A} \times 100 \%
$$

For small thresholds [Fig. 7(a)] the difference between the lasing modes and the matching QB states is very small. The average percent difference between all pairs of modes in this region is $\left\langle\sigma_{d}\right\rangle=4.2 \%$. For lasing modes with slightly larger thresholds [Fig. 7(b)] there are clear differences. Nevertheless, we may confidently match each lasing mode in this region with its corresponding QB state. The average percent difference between all pairs of modes in this region is $\left\langle\sigma_{d}\right\rangle=24 \%$. As mentioned above, it is challenging to find matching pairs of lasing modes and QB states for large thresholds. Figure 7(c) compares the lasing mode with the largest threshold and the QB state with the largest decay rate [circled in Fig. 6, region (c)]. Though these two modes are fairly close to each other in terms of $k, k_{0}^{\prime \prime}$, and $k^{\prime \prime}$, their intensity distributions are quite different. Indeed, there may be no correspondence between the two.

The deviation of the lasing modes from the QB states can be explained by the modification of the transfer matrix. In the passive system, $k_{0}^{\prime \prime}$ is constant, but $k^{\prime \prime} i=k_{0}^{\prime \prime} n(x)$ varies spatially. With the introduction of gain, $k^{\prime \prime}=k^{\prime \prime} n$ becomes constant within the random system, and feedback due to the inhomogeneity of $k^{\prime \prime}$ is removed. However, introducing gain generates additional feedback inside the 
random system caused by the modification in the real part of the wave vector $k^{\prime}=k n^{\prime}(x)$. Neglecting this effect results in some correspondence between lasing modes and QB states even at large thresholds [50]. Furthermore, since there is no gain outside the random system, $k^{\prime \prime}$ suddenly drops to zero at the system boundary. This discontinuity of $k^{\prime \prime}$ generates additional feedback for the lasing modes. In this weakly scattering system, the threshold gain is high. The large drop of $k^{\prime \prime}$ at the system boundary makes the additional feedback stronger.

\subsection{Two-Dimensional Random Lasers}

We turn now to the 2D case. A different approach based on the multipole method has been used. The multipole method is best suited to characterize multiple scattering problems involving scatterers with circular cross section. This method has been used to compute the scattering of a plane wave by a random collection of cylinders [30,51], to calculate the defect states in photonic crystals [52], to construct the exact Green's function of a finite system [53], or to calculate the local density of states [54]. This method has also been used to explain the anomalously large Lamb shift that occurs in photonic crystals by calculating the QB states in such structures [55]. Finally, the multipole method can be used to characterize the modes of 3D structures composed of cylinders [56] and in particular to find the modes of the photonic crystal fibers [57-59]. It will be used here to calculate the QB states and the lasing modes of the 2D disordered scattering systems of the kind shown in Fig. 1 and studied in the previous section for different regimes of scattering. Details about this method can be found in Appendix A.

This method is based essentially on a search for the poles of a scattering matrix. Because the system is open, the problem is not Hermitian, and hence there are no modes occurring for real wavelengths. The poles of the QB states all occur in the complex plane at wavelengths $\lambda=\lambda^{\prime}+i \lambda^{\prime \prime}$, with causality requiring that $\lambda^{\prime \prime}>0$. The real part of the wavelength $\lambda^{\prime}$ determines the resonance wavelength of the QB state, while the imaginary part $\lambda^{\prime \prime}$ determines the quality factor $Q$ $=\lambda^{\prime} /\left(2 \lambda^{\prime \prime}\right)$ of the mode [55].

The same method is used to find the lasing modes (TLM) at threshold. It is necessary this time to find the poles of the scattering matrix in the 2D space $\left(\lambda^{\prime}, \epsilon_{b}^{\prime \prime}\right)$ of real wavelengths $\left(\lambda^{\prime \prime}=0\right)$ and the imaginary component of the complex dielectric constant outside the scatterers where the gain is distributed. It can also be used to find the lasing modes when gain is localized inside of the scatterers. In this case the poles of the scattering matrix are searched in the space of real wavelengths $\left(\lambda^{\prime \prime}=0\right)$ and the imaginary part of the dielectric constant of cylinders $\epsilon_{l}^{\prime \prime}$.

The multipole method is both accurate and efficient: the boundary conditions are analytically satisfied, thus providing enhanced convergence, particularly when the refractive index contrast is high. However, in the case of large systems the method can be slow (given that field expansions are global, rather than local) when it is necessary to locate all poles within a sizable wavelength range. Another extremely efficient time-independent numerical method based on the finite element method [60] has been tested. This method is briefly described in Appendix B. We confirmed that the results obtained by both methods, the (purely numerical) finite element method and the (semi-analytic) multipole method were identical with a good precision.

\section{2a. Localized Case}

We first consider the localized case $\left(n_{l}^{\prime}=2.0\right)$ for which a complete comparison of the QB states and the lasing modes was possible with the time-dependent FDTD- 
$|\mathrm{E}|^{2}$

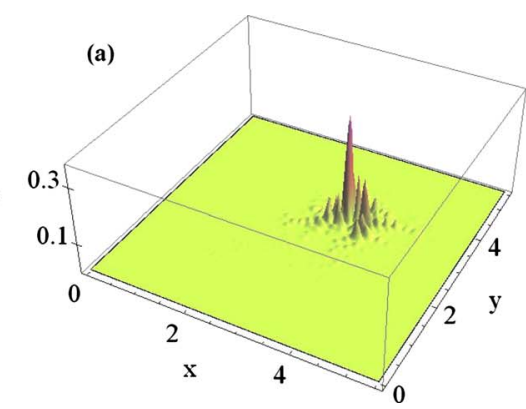

$|\mathrm{E}|^{2}$

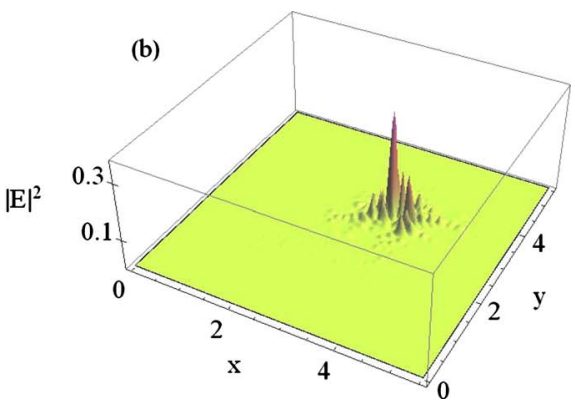

(a) Intensity $|E|^{2}$ of the localized QB state (Media 1) and (b) corresponding lasing mode (Media 2) calculated by using a multipole method for a 2D disordered scattering system of the kind shown in Fig. 1 with the refractive index of the cylinders $n_{l}^{\prime}=2.0$.

based method (Subsection 2.1), thus providing a reference comparison for the multipole calculations. The lasing mode is found at a wavelength $\lambda^{\prime}=446.335 \mathrm{~nm}$ for a value of the imaginary part of the refractive index $n_{l}^{\prime \prime}=-1.967 \times 10^{-4}$, representing the pumping threshold for this mode. The spatial distribution of its amplitude is shown in Fig. 8(b). The QB states of the passive system are calculated in the spectral vicinity of the lasing mode. The number of required multipoles was $N_{\max }=4$ (see Appendix A). Figure 8(a) shows the QB state that best resembles the lasing mode. Its wavelength and quality factor are, respectively, $\lambda^{\prime}=446.339 \mathrm{~nm}$ and $Q=8047$. The relative difference between the two modes is $\left\langle\sigma_{d}\right\rangle=0.05 \%$. These calculations provide confirmation that the lasing modes and the QB states are the same inside the scattering region for high- $Q$-valued states.

\section{2b. Diffusive Case}

We next consider the diffusive case and choose $n_{l}^{\prime}=1.25$. This is where the timeindependent method becomes interesting since, in contrast to the FDTD approach, it gives direct access to the QB states. They are accurately calculated in this regime for $N_{\max }=2$ multipoles. Figure 9 shows a lasing mode and its corresponding QB state.

Figure 9
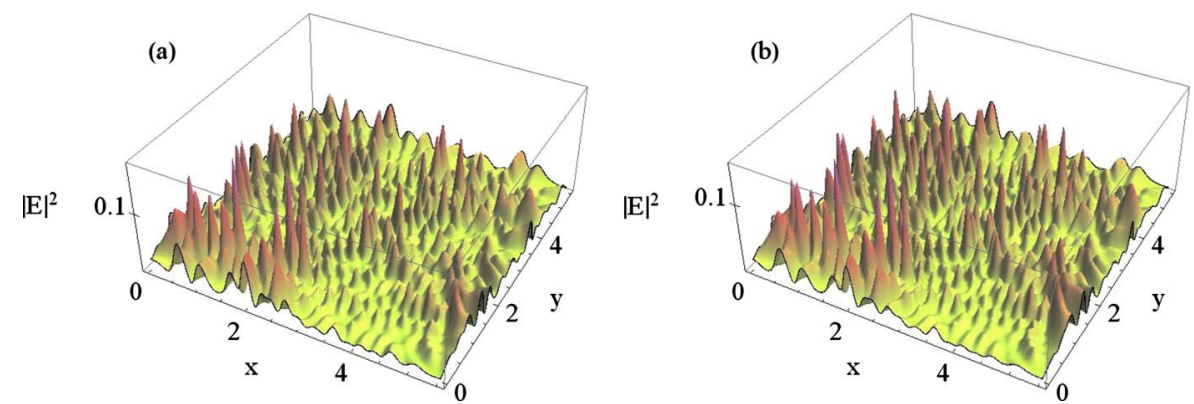

(a) Intensity $|E|^{2}$ of the diffusive QB state (Media 3) and (b) the lasing mode (Media 4) calculated by using the multipole method for the same random configuration as in Fig. 8 but with the refractive index of the cylinders of $n_{l}^{\prime}=1.25$. 
The lasing mode is found at $\lambda^{\prime}=455.827 \mathrm{~nm}$ for an imaginary part of the refractive index $n_{l}^{\prime \prime}=-3.778 \times 10^{-2}$. The wavelength and the quality factor of the QB state are, respectively, $\lambda^{\prime}=456.79 \mathrm{~nm}$ and $Q=29.2$. The lasing mode is therefore redshifted relative to the QB state's wavelength, as a result of the mode-pulling effect. The QB state and the lasing mode appear similar in Fig. 9. However, the relative difference between the two modes is larger than in the localized case, $\left\langle\sigma_{d}\right\rangle=14.5 \%$. Figure 10 shows the cross section of the spatial intensity of both modes along $x=2.75$. In spite of the resemblance, the two profile display visible dissimilarities. This suggests, in the diffusive case, that QB states and lasing modes are not exactly the same, though they exhibit quite similar features. These results are consistent with the findings presented in Subsection 2.2.

\section{2c. Transition Case}

It is both informative and interesting to follow the evolution of the lasing modes and the QB states spatial profile when the index of refraction is decreased progressively, allowing one to compare the QB states and the random lasing modes (TLM) systematically in a regime ranging from localized to diffusive. The QB state and lasing modes calculated for intermediate cylinder refractive indices $n_{l}^{\prime}=1.75$ and $n_{l}^{\prime}=1.5$ are displayed in Figs. 11 and 12 . We note that the highly spatially localized mode for $n_{l}^{\prime}=2$ (Fig. 8 ) is replaced for $n_{l}^{\prime}=1.75$ by a mode formed by two spatially localized peaks and several smaller peaks. For a refractive index of $n_{l}^{\prime}$ $=1.5$, the mode is still spatially localized, although in a larger area, but is now formed with a large number of overlapping peaks. A more systematic exploration of the nature of the lasing modes at the transition between localized states and extended resonances can be found in [29]. There, a scenario for the transition has been proposed based on the existence of necklace states which form chains of localized peaks, resulting from the coupling between localized modes. The modes shown here support this scenario. It is important to note that the decreasing scattering and increasing leakage not only affect the degree of spatial extension of the mode but also the nature of the QB states. Indeed, it was shown in [29] that, because of leakage, extended QB states have a nonvanishing imaginary part associated with a progressive component, in contrast to the purely stationary localized states. In Media1-4 we present animations of the time oscillation of the real part of the field

Figure 10

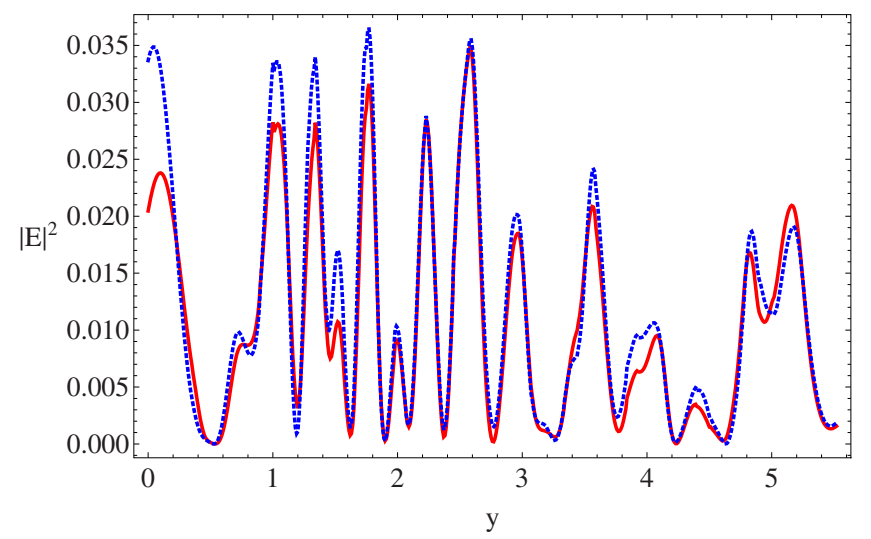

Intensity $|E|^{2}$ of the diffusive QB state (blue dashed curve) and lasing mode (red solid curve) for $x=2.75$ and $n_{l}^{\prime}=1.25$. 

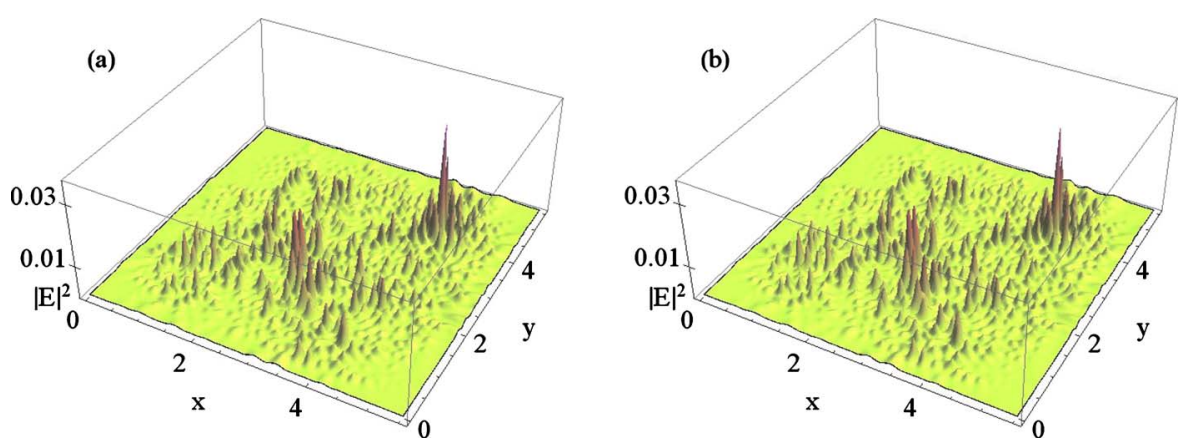

(a) Intensity $|E|^{2}$ of a QB state and (b) a lasing mode calculated by using multipole method for the same random configuration as above but with the refractive index of the cylinders $n_{l}^{\prime}=1.75$.

$\mathfrak{R}[\Psi \exp (-i \omega t)]$ of the QB state and of the corresponding TLM for $n^{\prime}=2$ and $n^{\prime}$ $=1.25$. The $\mathrm{QB}$ state is exponentially decaying in contrast to the lasing mode. The diffusive lasing mode clearly exhibit a progressive component, which does not exist in the localized lasing mode.

The values of wavelengths and quality factors of the QB states, lasing frequencies of the corresponding TLMs, and associated imaginary part of the refractive index are summarized in Table 1, together with the relative difference $\left\langle\sigma_{d}\right\rangle$ as defined in Eq. (9).

In order to visualize the increasing difference between TLM and QB states, the cross section of their spatial intensity profile at $x=2.75$ is plotted in Fig. 13. In Fig. 13(a) one cannot distinguish between the lasing mode and the QB state for $n^{\prime}$ $=1.75$, while for $n^{\prime}=1.5$ (Fig. 13(b)) differences begin to emerge, becoming more pronounced for the case of $n^{\prime}=1.25$ (Fig. 13(c)). This is seen also in the increase of the relative difference from $5 \%$ to $14.5 \%$. Clearly, there is a systematic increase of the discrepancy between QB states and lasing modes when index contrast and scattering decrease and leakage increases. For very low scattering $n^{\prime}=1.05$, we could not find the QB state corresponding to the TLM. Although we may have missed a pole in the complex plane, this raises, however, a serious question on the validity of the comparison of the threshold laser mode with QB states when weakly scattering

\section{Figure 12}
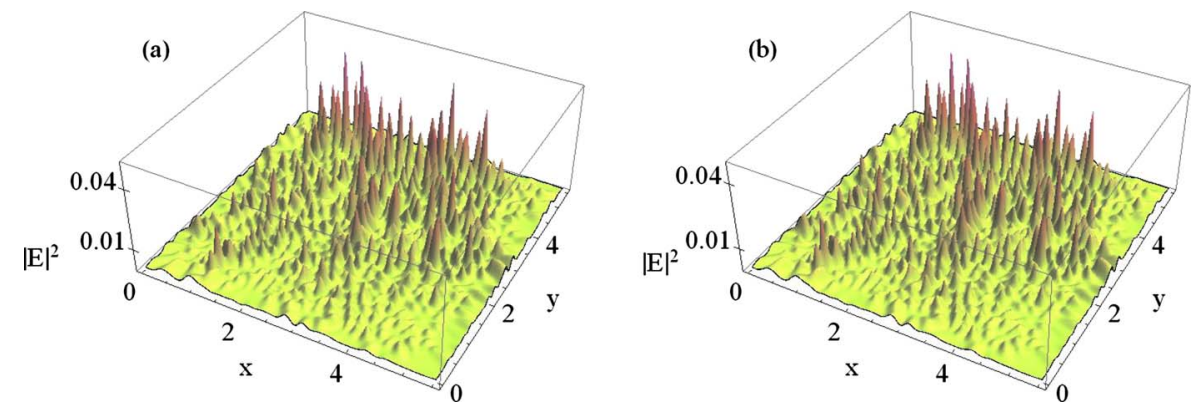

Same as in Fig. 11 but for $n_{l}^{\prime}=1.5$. 


\begin{tabular}{|c|c|c|c|c|}
\hline \multirow[b]{2}{*}{ Value } & \multicolumn{4}{|c|}{$n_{l}^{\prime}$} \\
\hline & 2.0 & 1.75 & 1.5 & 1.25 \\
\hline$\lambda^{\prime}(\mathrm{nm})(\mathrm{QB})$ & 446.339 & 451.60 & 456.60 & 456.79 \\
\hline$Q$ & 8047 & 161.28 & 87.8 & 29.2 \\
\hline$\lambda^{\prime}(\mathrm{nm})$ (laser) & 446.335 & 451.60 & 456.5 & 455.827 \\
\hline$n_{l}^{\prime \prime}$ & $-1.967 \times 10^{-4}$ & -0.0055 & -0.0124 & -0.0378 \\
\hline$\left\langle\sigma_{d}\right\rangle(\%)$ & 0.05 & 3 & 8.4 & 14.5 \\
\hline
\end{tabular}

${ }^{a}$ Wavelength $\lambda^{\prime}$ and quality factor $Q$ of the QB states; lasing frequency $\lambda^{\prime}$ and imaginary part of the refractive index $n_{l}^{\prime \prime}$ obtained for the threshold lasing modes; relative index difference $\left\langle\sigma_{d}\right\rangle$ between QB states and TLM.

\section{Figure 13}
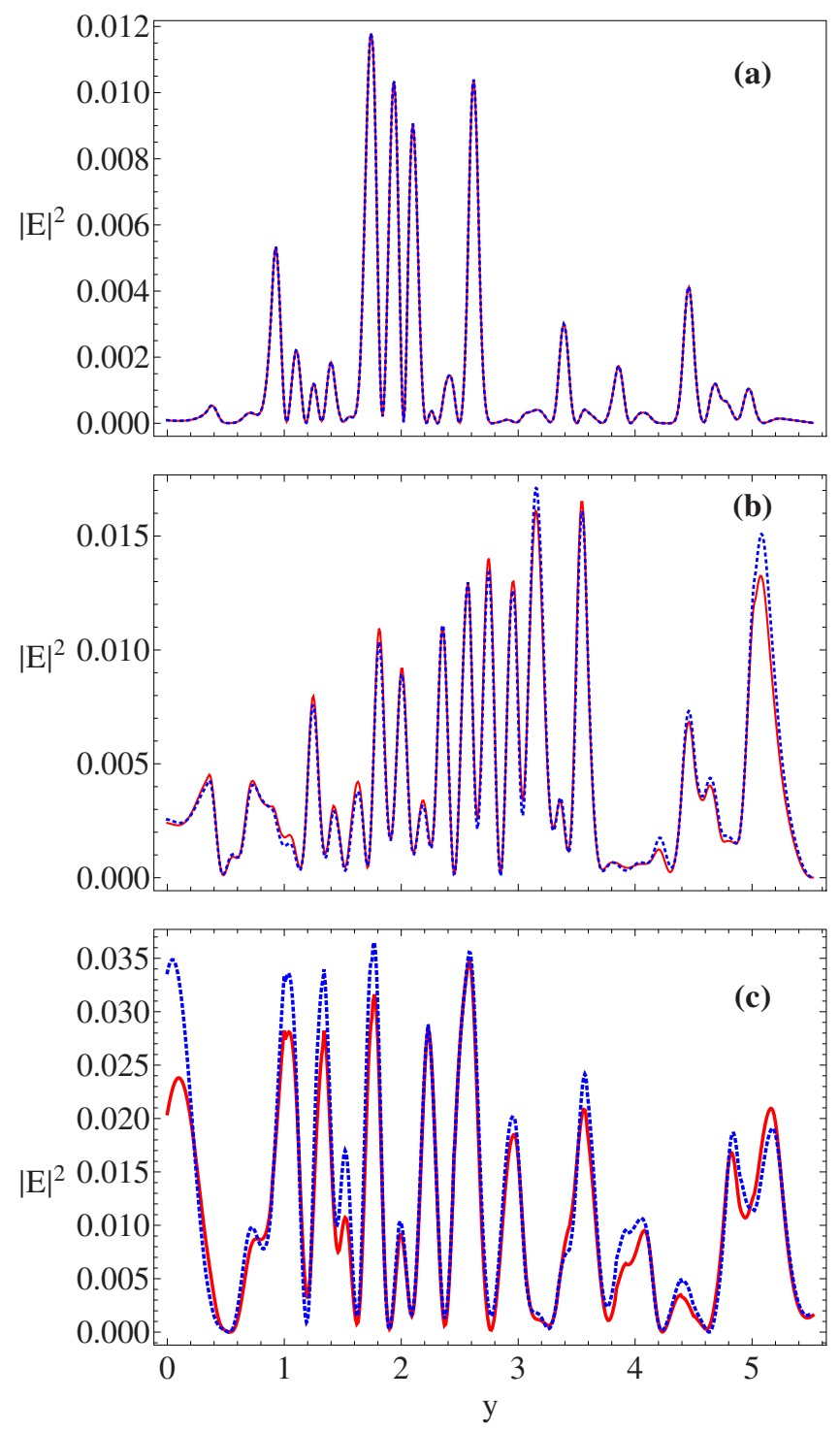

Intensity $|E|^{2}$ of QB state (blue dashed curves) and lasing mode (red solid curves) at $x=2.75$ for (a) $n_{l}^{\prime}=1.75$, (b) $n^{\prime}=1.5$, (c) $n^{\prime}=1.25$. 
systems are considered. In the next section we will argue that, in principle, QB states cannot be the support of the TLM. Section 5 will introduce a different class of states, which offer a valid basis on which the TLMs can be described.

\section{Threshold Lasing States versus Passive Cavity Resonances}

Semiclassical laser theory treats classical electromagnetic fields coupled to quantized matter and yields the thresholds, frequencies and electric fields of the lasing modes, but not their linewidths or noise properties. To treat the spatial dependence of lasing modes, one must go beyond rate equation descriptions and use the coupled nonlinear Maxwell-Bloch (MB) equations for light coupled to homogeneously broadened two-level atoms or multilevel generalizations thereof. These equations will be presented in Section 5 below. While the MB description has been used since the inception of laser theory $[61,62]$, in almost all cases simplifications to these equations were made, most notably a neglect of the openness of the laser cavity. As random lasers are strongly open systems, it is necessary to treat this aspect of the problem correctly to obtain a good description of them.

Historically a first breakthrough in describing Fabry-Perot type lasers with open sides was the Fox-Li method [44,45], which is an integral equation method of finding the passive cavity resonances of such a structure. It is widely assumed and stated that these resonances or QB states are the correct electromagnetic modes of a laser, at least at threshold. Often the nonlinear laser equations are studied with Hermitian cavity modes with phenomenological damping constants representing the cavity outcoupling loss obtained, e.g., from a Fox-Li calculation. It is worth noting that there are two kinds of cavity loss that occur in lasers; there is the outcoupling loss just mentioned and also the internal absorption of the cavity, which can be taken into account via the imaginary part of the passive cavity index of refraction. These are very different processes, as the former describes the usable coherent light energy emitted from the laser and the latter simply energy lost, usually as heat, in the laser cavity.

The QB states of an arbitrary passive cavity described by a linear dielectric function $\epsilon_{c}(\mathbf{x}, \omega)$ can be rigorously defined in terms of an electromagnetic scattering matrix $S$ for the cavity. This matrix relates incoming waves at wave vector $k$ (frequency $\omega=c k$ ) to outgoing waves in all of the asymptotic scattering channels and can be calculated from the wave equation. Note that while we speak of the frequency of the incoming wave, in fact the $S$ matrix is a time-independent quantity depending on the wave vector $k$. This is the wave vector outside the cavity; in random lasers we will be interested in spatially varying dielectric functions so that in the cavity there is no single wave vector of the field. For any laser, including the random laser, the cavity can be defined as simply the surface of last scattering, beyond which no backscattering occurs. The QB states are then the eigenvectors of the passive cavity $S$ matrix with eigenvalue equal to infinity; i.e., one has outgoing waves with no incoming waves. Because this boundary condition is incompatible with current conservation, these eigenvectors have the complex

wave vector $\tilde{k}_{\mu}$; these complex frequencies are the poles of the $S$ matrix and their imaginary parts must always be negative to satisfy causality conditions. There is normally a countably infinite set of such QB states. Because of their complex 
wave vector, asymptotically the QB states vary as $r^{-(d-1) / 2} \exp \left(+\left|\operatorname{Im}\left[\tilde{k}_{\mu}\right]\right| r\right)$ and diverge at infinity, so they are not normalizable solutions of the time-independent wave equation. Therefore we see that QB states cannot represent the lasing modes of the cavity, even at threshold, as the lasing modes have a real frequency and wave vector outside the cavity with conserved photon flux.

When gain is added to the cavity the effect is to add another contribution to the dielectric function $\epsilon_{g}(\mathbf{x}, \omega)$, which in general has a real and imaginary part. The imaginary part of $\epsilon_{g}$ has an amplifying sign when the gain medium is inverted and depends on the pump strength; it compensates for the outcoupling loss as well as any cavity loss from the cavity dielectric function $\epsilon_{c}$. The specific form of this function for the MB model will be given in Section 5 below. The TLMs are the solutions of the wave equation with $\epsilon_{\text {total }}(\mathbf{x})=\epsilon_{c}(\mathbf{x})+\epsilon_{g}(\mathbf{x})$ with only outgoing waves of real wave vector $k_{\mu}$ [we neglect henceforth for simplicity the frequency dependence of $\epsilon_{c}(\mathbf{x})$ ]. The $k_{\mu}$ are the wave vectors of the TLMs with real lasing frequencies $\Omega_{\mu}=c k_{\mu}$. These lasing wave vectors are clearly different from the complex $\tilde{k}_{\mu}$; moreover they are not equal to $\operatorname{Re}\left[\tilde{k}_{\mu}\right]$ as often supposed. This can be seen by the following continuity argument. Assume that $\epsilon_{c}(\mathbf{x})$ is purely real for simplicity, so that the $S$ matrix is unitary and all of its poles are complex and lie in the negative half-plane. Turn on the pump, which we will call $D_{0}$, anticipating our later notation, so that the inversion rises steadily from zero, continuously increasing the amplifying part of $\epsilon_{g}$. The $S$ matrix is no longer unitary, and its poles move continuously upward towards the real axis until each of them crosses the axis at a particular pump value, $D_{0}$ (see Fig. 14); the place where each pole crosses is the real lasing frequency $k_{\mu}$ for that particular TLM. Note that the poles do not move vertically to reach the real axis but always have some shift of the lasing frequency from the passive cavity frequency, mainly due to linepulling towards the gain center. As the $Q$ value of the cavity increases, the dis-

\section{Figure 14}

(a)

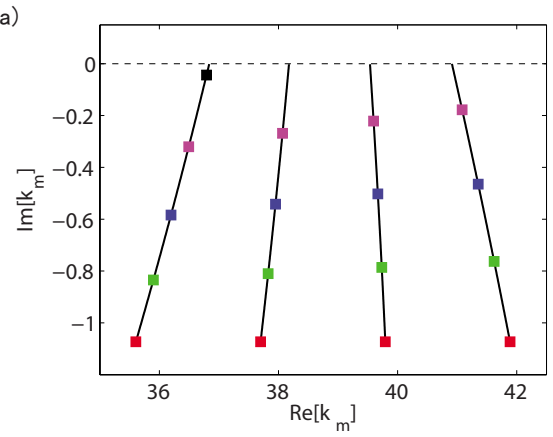

(b)

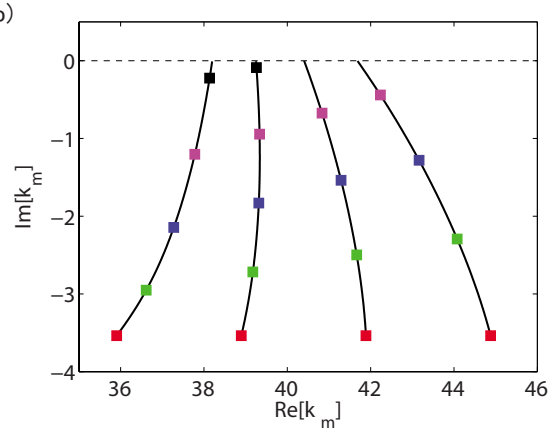

Shift of the poles of the $S$ matrix in the complex plane onto the real axis to form TLMs when the imaginary part of the dielectric function $\epsilon \equiv \epsilon_{c}+\epsilon_{g}$ varies for a simple 1D edge-emitting cavity laser [34]. The cavity is a region of length $L$ and uniform index (a) $n_{c}=1.5$, (b) $n=1.05\left(\epsilon_{c}=2.25,1.0025\right)$ terminated in vacuum at both ends. The calculations are based on the MB model discussed in Section 5, with parameters $k_{a} L=39$ and $\gamma_{\perp}=2$. (a) $n_{c}=1.5$; squares of different colors represent $\operatorname{Im}\left[\epsilon_{g}\right]=0,-0.032,-0.064,-0.096,-0.128$; (b) $n_{c}=1.05$; squares of different colors represent $\operatorname{Im}\left[\epsilon_{g}\right]=0,-0.04,-0.08,-0.12,-0.16$. Note the increase in the frequency shift in the complex plane for the leakier cavity. The center of the gain curve is at $k L=39$, which determines the visible line-pulling effect. 
tance the poles need to move to reach the real axis decreases, so that the frequency shift from $\operatorname{Re}\left[\tilde{k}_{\mu}\right]$ can become very small, and the conventional picture becomes more correct. In general the poles of the $S$ matrix are conserved quantities even in the presence of loss, so that the TLMs are in one-to-one correspondence with the QB states and thus are countably infinite, but for any cavity the pole that reaches the real axis first (i.e., at lowest pump $D_{0}$ ) is the actual first lasing mode. At higher pump values the nonlinear effects of saturation and mode competition will affect the behavior; so only the lowest-threshold TLM describes an observable lasing mode for fixed pumping conditions, the first lasing mode at threshold. Which pole gets there first depends not only on the $Q$ of the passive cavity resonance before gain is added, but also on the parameters of $\epsilon_{g}(\mathbf{x})$, which include the atomic transition frequency, the gain linewidth, and the pump conditions, as will be discussed below.

\section{Self-Consistent Time-Independent Approach to Random Lasing}

In Section 4 we gave a general argument based on the scattering matrix with the addition of gain to show that in general the QB states (passive cavity resonances) are never exactly the same as the TLMs, even inside the cavity. However the same argument indicated that inside a high- $Q$ cavity the two sets of functions become very similar, since the poles of the $S$ matrix are very close to the real axis and only a small amount of gain is required on order to move them to the real axis, which maps QB states onto TLMs. For localized states in the center of the sample the $Q$ values should be exponentially large and, as found numerically, QBs and TLMs should be indistinguishable (again, inside the cavity; outside the QB states have an unphysical growth). As already noted, the set of TLMs defines only threshold modes; as soon as the first TLM has turned on, it will alter the gain medium for the other potential modes through spatial hole burning, and a nonlinear approach needs to be considered. Very recently such an approach has been developed that has the major advantage of being time independent and partially analytic, providing both ease of computation and greater physical insight. The approach, due to Türeci-Stone-Ge, is known as steady-state ab initio laser theory (SALT) [31,34,35]. It finds the stationary solutions of the MB semiclassical lasing equations in the multimode regime, for cavities of arbitrary complexity and openness, and to infinite order in the nonlinear interactions. As such it is ideal for treating diffusive or quasi-ballistic random lasers, which are extremely open and typically highly multimode even slightly above threshold. In this section we present the basic ideas with emphasis on TLMs, which are the focus of this review. The nonlinear theory has been reviewed in some detail elsewhere [35], and we just present a brief introduction to it here.

\subsection{Maxwell-Bloch Threshold Lasing Modes}

The MB semiclassical laser equations describe a gain medium of identical twolevel atoms with energy level spacing $\hbar \omega_{a}=\hbar c k_{a}$ and relaxation rate $\gamma_{\|}$, being pumped by an external energy source, $D_{0}$ (which can vary in space), contained in a cavity that can be described by a linear dielectric function, $\epsilon_{c}(\mathbf{x})$. This leads to a population inversion of the atoms, $D(\mathbf{x}, t)$, which in the presence of an electric field creates a nonlinear polarization of the atomic medium, $P(\mathbf{x}, t)$, which itself 
is coupled nonlinearly to the inversion through the electric field, $E(\mathbf{x}, t)$. The electric field and the nonlinear polarization are related linearly through Maxwell's wave equation, although above the first lasing threshold the polarization is implicitly a nonlinear function of the electric field. The induced polarization also relaxes at a rate $\gamma_{\perp}$ that is typically much greater than the rate $\gamma_{\|}$at which the inversion relaxes, and this is a key assumption in our treatment of the nonlinear regime, but will not be needed in the initial discussion of TLMs.

The resulting system of nonlinear coupled partial differential equations for the three fields $E(\mathbf{x}, t), P(\mathbf{x}, t), D(\mathbf{x}, t)$ are $(c=1)$

$$
\begin{aligned}
& \ddot{E}^{+}=\frac{1}{\epsilon_{c}(\mathbf{x})} \nabla^{2} E^{+}-\frac{4 \pi}{\epsilon_{c}(\mathbf{x})} \ddot{P}^{+}, \\
& \dot{P}^{+}=-\left(i \omega_{a}+\gamma_{\perp}\right) P^{+}+\frac{g^{2}}{i \hbar} E^{+} D, \\
& \dot{D}=\gamma_{\|}\left(D_{0}-D\right)-\frac{2}{i \hbar}\left(E^{+}\left(P^{+}\right)^{*}-P^{+}\left(E^{+}\right)^{*}\right) .
\end{aligned}
$$

Here $g$ is the dipole matrix element of the atoms, and the units for the pump are chosen so that $D_{0}$ is equal to the time-independent inversion of the atomic system in the absence of an electric field. This pump can be nonuniform: $D_{0}$ $=D_{0}(\mathbf{x})$ based on the experimental pump conditions, but we will not discuss that case here. The electric field, polarization, and inversion are real functions ( $E, P$ are vector functions in general, but we assume a geometry where they can be treated as scalars). In writing the equations above we have written these fields in the usual manner in terms of their positive and negative frequency components, $E=E^{+}+E^{-}, P=P^{+}+P^{-}$, and then made the rotating wave approximation in which the coupling of negative to positive components is neglected. There is no advantage in our treatment to making the standard slowly varying envelope approximation, and we do not make it.

\subsection{Self-Consistent Steady-State Lasing Equations}

The starting point of our formulation is to assume that there exists a steady-state multiperiodic solution of Eqs. (10)-(12) above; i.e., we try a solution of the form

$$
E^{+}(\mathbf{x}, t)=\sum_{\mu=1}^{N} \Psi_{\mu}(\mathbf{x}) e^{-i k_{\mu} t}, \quad P^{+}(\mathbf{x}, t)=\sum_{\mu=1}^{N} P_{\mu}(\mathbf{x}) e^{-i k_{\mu} t} .
$$

Having taken $c=1$ we do not distinguish between frequency and wave vector. The functions $\Psi_{\mu}(\mathbf{x})$ are the unknown lasing modes, and the real numbers $k_{\mu}$ are the unknown lasing frequencies; these functions and frequencies are not assumed to have any simple relationship to the QB states of the passive cavity and will be determined self-consistently. As the pump increases from zero the number of terms in the sum will vary, $N=0,1,2, \ldots$; at a series of thresholds each new mode will appear. The general nonlinear theory is based on a self-consistent equation that determines how many modes there are at a given pump and solves for these modes and their frequencies. However in this section we will discuss TLMs, and so we need only consider one term in the sum. Furthermore, at the 
first threshold the electric field is negligibly small, and so the inversion is equal to the external pump profile, assumed uniform in space, $D(\mathbf{x}, t)=D_{0}$. Assuming single-mode lasing, the equation for the polarization becomes

$$
P_{\mu}(\mathbf{x})=\frac{-i D_{0} g^{2} \Psi_{\mu}(\mathbf{x})}{\hbar\left(\gamma_{\perp}-i\left(k_{\mu}-k_{a}\right)\right)} .
$$

Having found $P_{\mu}(\mathbf{x})$ in terms of $\Psi_{\mu}(\mathbf{x}), D_{0}$, we substitute this result into the righthand side of Maxwell's equation along with $\Psi_{\mu}(\mathbf{x})$ for the electric field on the left-hand side. The result is

$$
\left[\nabla^{2}+\epsilon_{c}(\mathbf{x}) k_{\mu}^{2}\right] \Psi_{\mu}(\mathbf{x})=\frac{i D_{0} 4 \pi g^{2} k_{\mu}^{2} \Psi_{\mu}(\mathbf{x})}{\hbar\left(\gamma_{\perp}-i\left(k_{\mu}-k_{a}\right)\right)},
$$

which can be written in the form

$$
\left[\nabla^{2}+\left(\epsilon_{c}(\mathbf{x})+\epsilon_{g}(\mathbf{x})\right) k_{\mu}^{2}\right] \Psi_{\mu}(\mathbf{x})=0
$$

where $\epsilon_{g}(\mathbf{x})$ is the dielectric function of the gain medium, which only varies in space if the external pump or the gain atoms are nonuniform. Defining convenient units of the pump $D_{0 c}=\hbar \gamma_{\perp} / 4 \pi k_{a}^{2} g^{2}$ and replacing $D_{0} \Rightarrow D_{0} / D_{0 c}$, we find that

$$
\epsilon_{g}(\mathbf{x})=\frac{D_{0}}{k_{a}^{2}}\left[\frac{\gamma_{\perp}\left(k_{\mu}-k_{a}\right)}{\gamma_{\perp}^{2}+\left(k_{\mu}-k_{a}\right)^{2}}+\frac{-i \gamma_{\perp}^{2}}{\gamma_{\perp}^{2}+\left(k_{\mu}-k_{a}\right)^{2}}\right] .
$$

Equation (16) is to be solved with the boundary condition that at infinity one has only an outgoing wave at frequency $k_{\mu}$, i.e., $\nabla_{r} \Psi_{\mu}(\mathbf{x})=+i k_{\mu} \Psi_{\mu}(\mathbf{x})$ when $r \rightarrow \infty$. In general this equation with this boundary condition cannot be solved for an arbitrary choice of the lasing frequency $k_{\mu}$ and for arbitrary values of the pump $D_{0}$; it is necessary to vary $k_{\mu}$ and the pump strength $D_{0}$ to find the countably infinite set of values $\left(k_{\mu}, D_{0}^{(\mu)}\right)$ at which a solution exists. This variation is equivalent to the pulling of the $S$-matrix poles onto the real axis discussed in Section 4 above; $D_{0}^{(\mu)}$ defines the threshold pump for that pole, and $k_{\mu}$ the point at which it crosses the real axis. As noted, while all of these solutions can be classified as TLMs, only the solution with the lowest value of $D_{0}^{(\mu)}$ will actually be a physical lasing state, as higher lasing modes are altered by nonlinear modal interactions.

Equation (16) shows that the TLMs are the solutions of the original Maxwell equation with the addition of a complex, pump- and frequency-dependent dielectric function that is uniform in space (for the assumed uniform pumping). The imaginary and the real parts of the gain dielectric function have the familiar symmetric and antisymmetric two-level resonance forms, respectively. The dependence on the atomic frequency $k_{a}$ encodes the usual atomic line-pulling effect. In the limit of a very broad gain curve $\left(\gamma_{\perp} \rightarrow \infty\right)$ the line-pulling effects can be neglected, and we find the simple result

$$
\epsilon_{g} \rightarrow-i D_{0} / k_{a}^{2},
$$

i.e., a constant imaginary (amplifying) part of $\epsilon_{g}$ proportional to the pump strength. Such linear gain models have been studied before, although typically with a constant imaginary part of the index of refraction instead of a constant imaginary part of the dielectric function. Our results show that, in order to reproduce the TLMs of the MB equations, one needs to take 


$$
n(\mathbf{x})=\sqrt{\epsilon_{c}(\mathbf{x})+\epsilon_{g}\left(D_{0}, k_{\mu}-k_{a}, \gamma_{\perp}\right)}
$$

so that the pump changes both the real and the imaginary parts of the index of refraction.

\subsection{Solution for Threshold Lasing Modes and Constant-Flux States}

The differential equation (16) is self-consistent in the sense that the boundary conditions depend on the eigenvalue $k_{\mu}$ that one is solving for, and so some form of nonlinear search is required. The required search turns out to be much more convenient if one writes an equivalent integral form of the equation transforming it into a self-consistent eigenvalue problem. For this purpose we rewrite it in the form

$$
\left[\epsilon_{c}(\mathbf{x})^{-1} \nabla^{2}+k_{\mu}^{2}\right] \Psi_{\mu}(\mathbf{x})=\frac{-\epsilon_{g} k_{\mu}^{2}}{\epsilon_{c}(\mathbf{x})} \Psi_{\mu}(\mathbf{x})
$$

and then, treating the right-hand side as a source, invert the equation with the appropriate Green function to obtain

$$
\Psi_{\mu}(\mathbf{x})=\frac{i D_{0} \gamma_{\perp}}{\gamma_{\perp}-i\left(k_{\mu}-k_{a}\right)} \frac{k_{\mu}^{2}}{k_{a}^{2}} \int_{\mathcal{D}} d \mathbf{x}^{\prime} \frac{G\left(\mathbf{x}, \mathbf{x}^{\prime} ; k_{\mu}\right) \Psi_{\mu}\left(\mathbf{x}^{\prime}\right)}{\epsilon_{c}\left(\mathbf{x}^{\prime}\right)}
$$

Here the integral is over the gain region, which we will assume coincides with the cavity region $\mathcal{D}$. The appropriate Green function satisfies

$$
\left[\epsilon_{c}(\mathbf{x})^{-1} \nabla^{2}+k^{2}\right] G\left(\mathbf{x}, \mathbf{x}^{\prime} \mid k\right)=\delta^{d}\left(\mathbf{x}-\mathbf{x}^{\prime}\right)
$$

and is non-Hermitian because of the outgoing wave boundary conditions: $\left.\nabla_{r} G\left(\mathbf{x}, \mathbf{x}^{\prime} \mid k\right)\right|_{r \rightarrow \infty}=\left.\nabla_{r^{\prime}} G\left(\mathbf{x}, \mathbf{x}^{\prime} \mid k\right)\right|_{r^{\prime} \rightarrow \infty}=i k G\left(\mathbf{x}, \mathbf{x}^{\prime} \mid k\right)$, where $\nabla_{r}$ is the radial derivative. $G\left(\mathbf{x}, \mathbf{x}^{\prime} \mid k\right)$ has the spectral representation

$$
G\left(\mathbf{x}, \mathbf{x}^{\prime} \mid k\right)=\sum_{m} \frac{\varphi_{m}(\mathbf{x}, k) \bar{\varphi}_{m}^{*}\left(\mathbf{x}^{\prime}, k\right)}{\left(k^{2}-k_{m}^{2}\right)} .
$$

We refer to the functions $\varphi_{m}(\mathbf{x}, k)$ in Eq. (23) as the CF states. They satisfy

$$
\left[\epsilon_{c}(\mathbf{x})^{-1} \nabla^{2}+k_{m}^{2}\right] \varphi_{m}(\mathbf{x}, k)=0
$$

with the corresponding non-Hermitian boundary condition of purely outgoing spherical waves of fixed frequency $k$ (eventually set equal to the lasing frequency) at infinity. Their dual (biorthogonal) partners $\bar{\varphi}_{m}\left(\mathbf{x}^{\prime}, k\right)$ satisfy the complex conjugate differential equation with purely incoming wave boundary conditions. These dual sets satisfy the biorthogonality relation

$$
\int_{\mathcal{D}} d \mathbf{x} \varphi_{m}(\mathbf{x}, k) \bar{\varphi}_{n}^{*}(\mathbf{x}, k)=\delta_{m n}
$$

with appropriate normalization.

The CF states satisfy the standard wave equation, Eq. (24), but with the nonHermitian boundary condition already mentioned; hence their eigenvalues $k_{m}^{2}$ are complex, with (it can be shown) a negative imaginary part, corresponding to 
amplification within the cavity. However, outside the cavity, by construction, they have the real wave vector $k_{\mu}$ and a conserved photon flux. They are a complete basis set for each lasing frequency $k_{\mu}$, and hence they are a natural choice to represent the TLMs as well as the lasing modes above threshold. Hence we make the expansion

$$
\Psi_{\mu}(\mathbf{x})=\sum_{m=1}^{\infty} a_{m}^{\mu} \varphi_{m}^{\mu}(\mathbf{x}) .
$$

Substituting this expansion into Eq. (21), using biorthogonality, and truncating the expansion at $N$ terms, leads to the eigenvalue problem

$$
a_{m}^{\mu}=D_{0} \Lambda_{m}\left(k_{\mu}\right) \int_{\mathcal{D}} d \mathbf{x}^{\prime} \frac{\bar{\varphi}_{m}^{\mu *}\left(\mathbf{x}^{\prime}\right) \sum_{p}^{N} a_{p}^{\mu} \varphi_{p}^{\mu}\left(\mathbf{x}^{\prime}\right)}{\epsilon_{c}\left(\mathbf{x}^{\prime}\right)} \equiv D_{0} \sum_{p}^{N} \mathcal{T}_{m p}^{(0)} a_{p}^{\mu},
$$

where $\Lambda_{m}(k) \equiv i \gamma_{\perp}\left(k^{2} / k_{a}^{2}\right) /\left[\left(\gamma_{\perp}-i\left(k-k_{a}\right)\right)\left(k^{2}-k_{m}^{2}(k)\right)\right]$.

One sees that the TLMs in the CF basis are determined by the condition that an eigenvalue of the matrix $D_{0} \mathcal{T}^{(0)}\left(k_{\mu}\right)$ is equal to unity. Since the matrix $\mathcal{T}^{(0)}\left(k_{\mu}\right)$ is independent of $D_{0}$, it is natural to focus on this object, which we call the threshold matrix. It is a complex matrix with no special symmetries, implying that its eigenvalues $\lambda_{\mu}$ are all complex for a general value of $k_{\mu}$. If the real control parameter $D_{0}$ (the pump) is set equal to $1 /\left|\lambda_{\mu}\right|$, then the matrix $D_{0} \mathcal{T}^{(0)}\left(k_{\mu}\right)$ will have an eigenvalue of modulus unity, but not a real eigenvalue equal to unity as required, and no solution for the TLMs exists for this choice of $k_{\mu}$. It is the phase condition that $\lambda_{\mu}\left(k_{\mu}\right)$ must be real that determines the allowed lasing frequencies. In practice one orders the $\lambda_{\mu}$ in decreasing modulus based on an initial approximation to the lasing frequency, $k_{\mu}$, and then tunes $k_{\mu}$ slowly until each eigenvalue flows through the real axis [which is guaranteed by the dominant $k$ dependence contained in the factor $\Lambda_{m}(k)$ ]. Normally the eigenvalues do not switch order during this flow, and the largest eigenvalue $\lambda_{\mu}$ will determine the lowest threshold TLM, with threshold $D_{0}^{(\mu)}=1 / \lambda_{\mu}\left(k_{\mu}\right)$, where $k_{\mu}$ is the frequency that makes the largest eigenvalue $\mathcal{T}^{(0)}\left(k_{\mu}\right)$ real. The eigenvector corresponding to $\lambda_{\mu}$ gives the coefficients for the CF expansion of the TLM of the first mode $\Psi_{\mu}(\mathbf{x})$. TLMs with higher thresholds can be found by imposing the reality condition on smaller eigenvalues of $\mathcal{T}^{(0)}\left(k_{\mu}\right)$. This approach has been described in detail elsewhere [33,35], and provides a much more efficient method for finding TLMs than solving the self-consistent differential equation, Eq. (16).

We immediately see from Eqs. (25) and (27) that for an arbitrarily shaped cavity of uniform dielectric constant $\epsilon_{c}$ the matrix $\mathcal{T}^{(0)}\left(k_{\mu}\right)$ is diagonal owing to the biorthogonality of the CF states. Thus each TLM is a single CF state, corresponding to one of the $k_{\mu}$ that satisfies the reality condition. In this case the expansion of $\Psi_{\mu}(\mathbf{x})$ consists of just one term, and the threshold lasing equation is equivalent to Eq. (24) with appropriate relabeling. When $\epsilon_{c}$ varies in space, as for random lasers, the threshold matrix is not diagonal, and there can in principle be many CF states contributing to one TLM [63]. However, since $\varphi_{m}(\mathbf{x}), \bar{\varphi}_{p}(\mathbf{x})$ are uncorrelated fluctuating functions of space, it turns out that the threshold matrix in random lasers is approximately diagonal and the threshold modes are dominated by one, pseudorandom CF state determined by solving Eq. (24) for the appropriate random dielectric function $\epsilon_{c}(\mathbf{x})$. This is shown in Fig. 15. In summary, the theory leading to the threshold equation (27) gives an efficient time-independent 


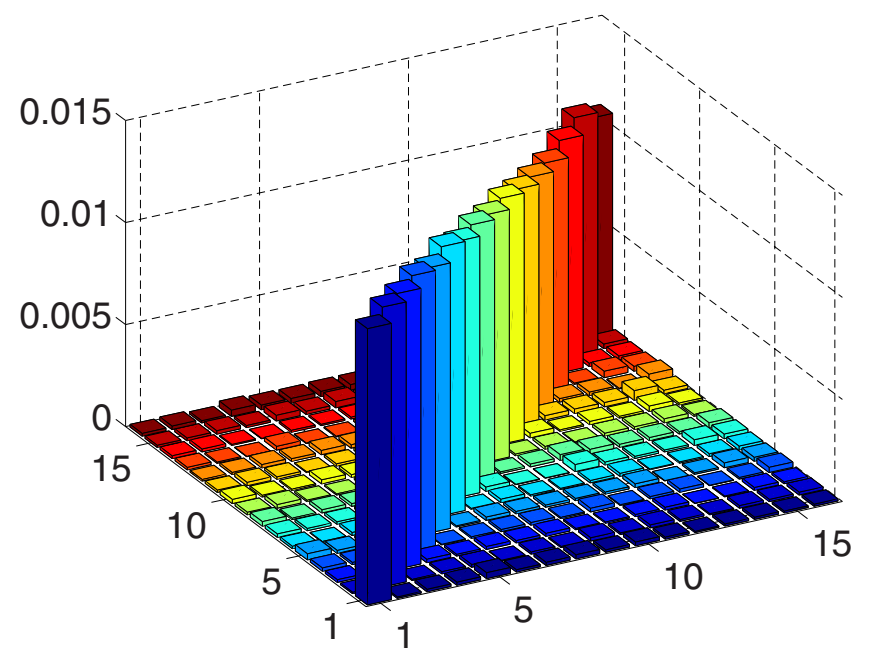

Typical values of the threshold matrix elements $\mathcal{T}^{(0)}$ in a $2 \mathrm{D}$ random laser schematized in the inset of Fig. 18 below, using sixteen CF states. The off-diagonal elements are one to two orders of magnitude smaller than the diagonal ones.

method for finding the TLMs of random lasers in any disorder regime. In general these TLMs are very close to a single CF state determined by Eq. (24) at the lasing frequency $k_{\mu}$. With this new method TLMs of random lasers can be found for complex 2D and even 3D geometries. In Figs. 16 and 17 we compare TLMs, CF states and QB states for the 2D random laser model used in [33], illustrating the agreement of TLMs with CF states even for weak scattering, while a significant deviation from the closest QB state is found.

This SALT is well-suited to describe not just TLMs but to find the true multimode lasing spectrum of random lasers above threshold. This will not be treated in detail here, but in the next section we briefly explain the basic approach in the nonlinear theory and show one representative result.

\subsection{Nonlinear Steady-State ab Initio Laser Theory}

The key to generalizing SALT to the multimode nonlinear regime is to return to the fundamental $\mathrm{MB}$ equations and go beyond the assumption that the inversion $D(\mathbf{x}, t)$ is equal to the constant threshold pump $D_{0}$. Once lasing modes have turned on, their spatially varying electric fields cause varying degrees of stimulated emission from the gain atoms and hence tend to reduce the inversion $D$ from the pump value $D_{0}$ in a manner that varies in space and in principle in time. However it has been shown that if $\gamma_{\perp} \gg \gamma_{\|}$, then the time dependence of the inversion is weak, and although $D$ varies in space, it is a good approximation to take $D(\mathbf{x}, t)=D(\mathbf{x})$. This stationary inversion approximation has been used in laser theory for many years, going back to Haken [62], but has not been incorporated into an ab initio method such as SALT. We will not review the details of the derivation of the nonlinear multimode theory of Türeci-Stone-Ge, which have been given elsewhere $[31,35]$. Instead we just state that the net effect of the nonlinear interactions within the stationary inversion approximation is just to replace the uniform inversion, as follows, 
(a)

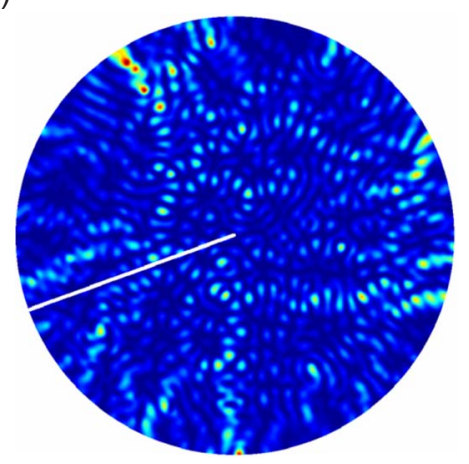

(c)

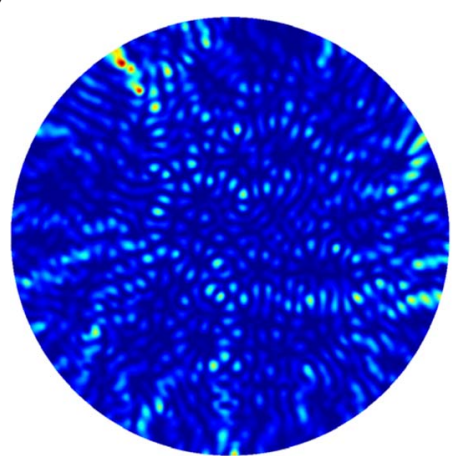

(b)

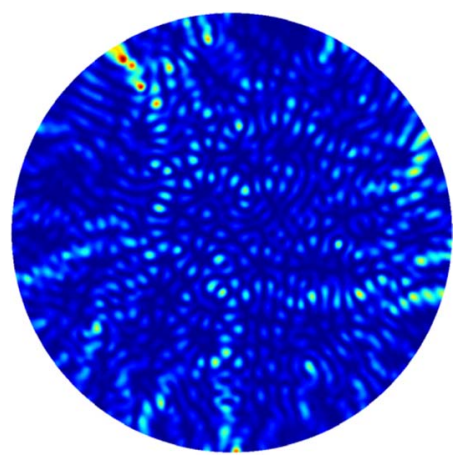

(d)

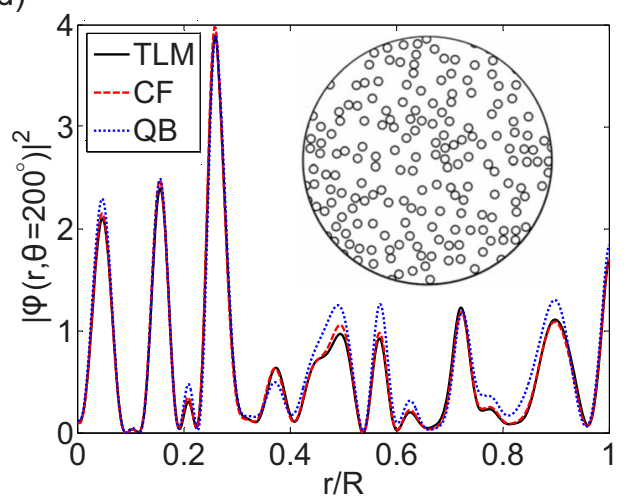

(a) False color plot of one TLM in a 2D random laser modeled as an aggregate of subwavelength particles of index of refraction $n=1.2$ and radius $r=R / 30$ against a background index $n=1$ imbedded in a uniform disk of gain material of radius $R$ [see inset, panel (d)]. The frequency of the lasing mode is $k R=59.9432$, which is pulled from (b) the real part of the dominating CF state $k_{m} R=59.8766-0.8593 i$ towards the transition frequency $k_{a} R=60$. The spatial profile of the TLM and CF state agree very well, whereas (c) the corresponding QB state $\tilde{k}_{m} R=59.8602-0.8660 i$ differs from that of the TLM and the CF state noticeably, as can be seen in (d), where we plot the internal intensity along the $\theta=200^{\circ}$ direction [white line in (a)].

$$
D_{0} \rightarrow \frac{D_{0}}{\left.1+\sum_{\nu} \Gamma\left(k_{\nu}\right)\left|\Psi_{\nu}(\mathbf{x})\right|^{2}\right)}
$$

in all of the equations of the theory of the TLMs. Here $\nu$ labels all abovethreshold modes and $\Gamma\left(k_{\nu}\right)$ is a Lorentzian centered at the lasing frequency of mode $\nu$ with width $\gamma_{\perp}$. If we make this substitution into Eq. (21), we arrive at the fundamental integral equation of SALT:

$$
\Psi_{\mu}(\mathbf{x})=\frac{i D_{0} \gamma_{\perp}}{\gamma_{\perp}-i\left(k_{\mu}-k_{a}\right)} \frac{k_{\mu}^{2}}{k_{a}^{2}} \int_{\mathcal{D}} d \mathbf{x}^{\prime} \frac{G\left(\mathbf{x}, \mathbf{x}^{\prime} ; k_{\mu}\right) \Psi_{\mu}\left(\mathbf{x}^{\prime}\right)}{\epsilon_{c}\left(\mathbf{x}^{\prime}\right)\left(1+\sum_{\nu} \Gamma_{\nu}\left|\Psi_{\nu}\left(\mathbf{x}^{\prime}\right)\right|^{2}\right)}
$$

Note that this equation shows that each lasing mode interacts with itself (saturation) and all other lasing modes (mode competition) via the hole-burning de- 
(a)

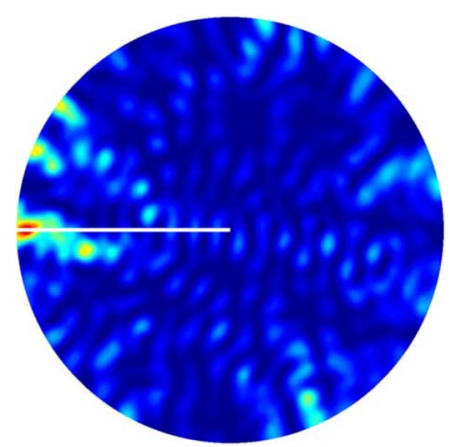

(c)

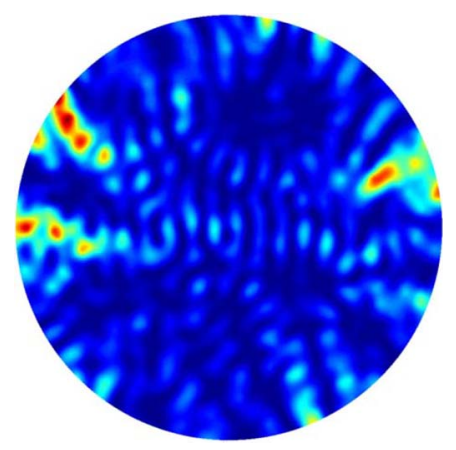

(b)

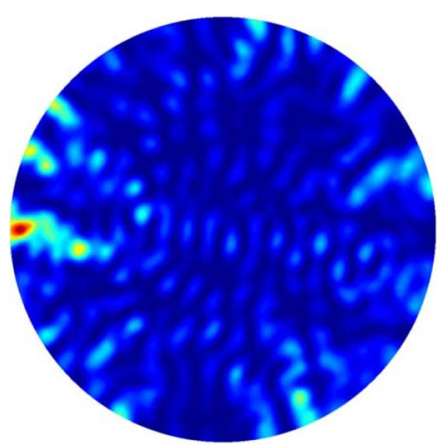

(d)

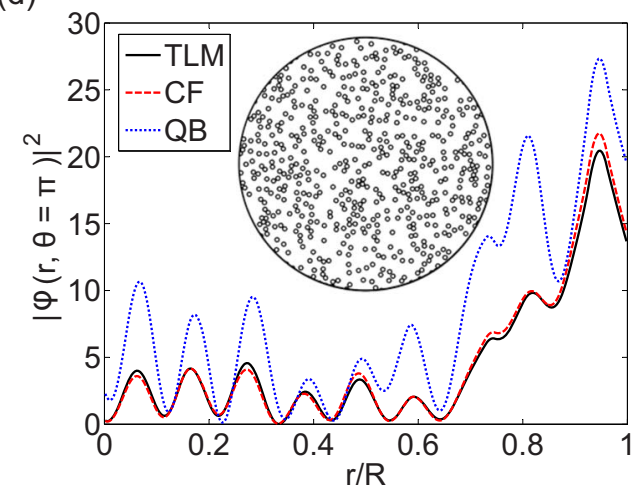

(a) False color plot of one TLM in a 2D random laser similar to that in Fig. 16 but with particles of radius $r=R / 60$, corresponding to weaker scattering [see inset, panel (d)]. The frequency of the lasing mode is $k R=29.9959$, which is very close to (b) the CF state $k_{m} R=30.0058-1.3219 i$ but shifted from (c) the corresponding QB state $\tilde{k}_{m} R=29.8813-1.3790 i$. (d) Internal intensity of the three states in the $\theta=\pi$ direction [white line in (a)]; because of weaker scattering the QB state now differs substantially from the CF and TLM, which still agree quite well with each other.

nominator of Eq. (28). This set of coupled nonlinear equations is still conveniently solved in the basis of CF states for each modal frequency $k_{\mu}$, as for the TLMs; the details have been given elsewhere $[33,35]$.

The first results of the SALT for the modal properties of multimode random lasers in weak-scattering 2D media were given in [33]. We will not present a full picture of these results here, but just show some properties of the random laser lasing frequencies in Fig. 18. The model is explained in the figure caption (see inset). The complex CF and QB frequencies are shown to be distinct, and the lasing frequencies are subject to very strong line-pulling effects.

The new tool of SALT allows one to study random lasers with full nonlinear interactions in $2 \mathrm{D}$ and even in $3 \mathrm{D}$. The elimination of time dependence in this theory makes larger and more complex cavities computationally tractable. The theory also provides a new language based on CF states to describe the lasing modes. Now detailed statistical studies as well as comparisons to statistical models based on random matrix theory, disordered media theory, and wave chaos theory are needed. Such studies are in progress. 

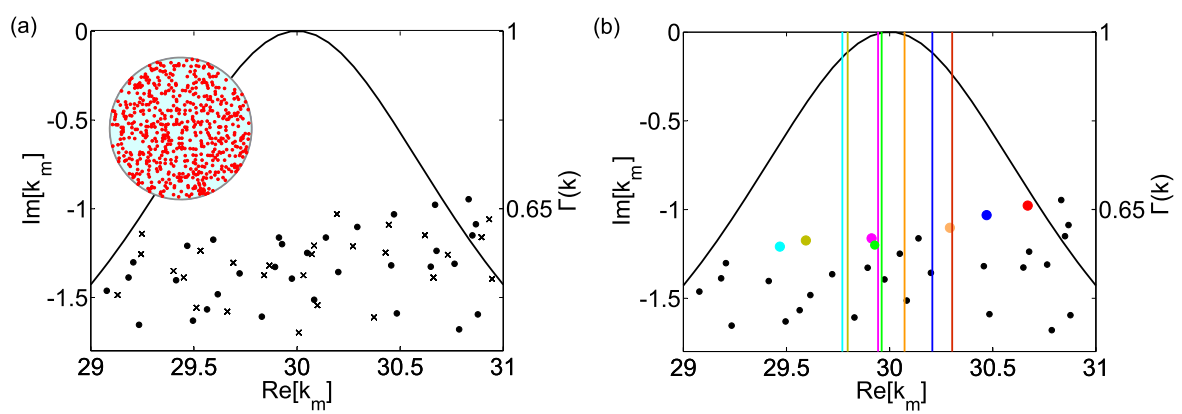

(a) CF (dots) and QB (crosses) frequencies in a 2D random laser modeled as an aggregate of subwavelength particles of index of refraction $n=1.2$ against a background index $n=1$ imbedded in a uniform disk of gain material (see inset). The two sets of complex frequencies are statistically similar but differ substantially. The solid curve shows the gain curve $\Gamma(k)$ with $\gamma_{\perp}=1$. (b) Lasing frequencies of the same random system well above threshold (colored lines). Colored circles denote the CF state dominating the correspondingly colored modes at threshold.

\section{Conclusion}

A decade of theoretical study of random lasers has clarified the nature of the lasing modes in disordered systems with multiple scattering and gain. Most important, it has been established that high- $Q$ passive cavity modes such as those created by Anderson localization or by rare fluctuations of various kinds are not necessary in order to have self-organized laser oscillation at a frequency distinct from the atomic transition frequency (gain center). In addition, this study has emphasized a point of general importance in laser theory, that TLMs are not identical to the QB states (resonances) of the passive cavity. This point is demonstrated by a number of numerical calculations presented above and also can be understood from the realization that the QB states are eigenvectors of the unitary $S$ matrix of the cavity without gain, but at complex frequency, whereas the TLMs are eigenvectors of the nonunitary $S$ matrix of the cavity with gain and with real frequency. The difference between these eigenvectors (within the cavity), which is large in the weak scattering limit, becomes small in the diffusive regime as the $Q$ of the cavity increases and is negligible, e.g., for Anderson localized modes and for high- $Q$ modes of conventional cavities. The new basis set of constant flux (CF) states provides a better approximation for finding the TLMs of random lasers and coincides with the exact lasing modes of uniform index cavities. Further statistical and analytical study is necessary to characterize the properties of random lasers in the different regimes, weak scattering, diffusive, and localized, and to understand the effects of nonlinear interactions.

\section{Appendix A: Multipole Method}

This appendix details the principle of the multipole method as used in this paper and its implementation. Although we describe here the method for 2D systems, it can be also applied to 3D structures.

We consider a random collection of $N_{c}$ nonoverlapping cylinders with arbitrary complex dielectric constant $\epsilon_{l}=\epsilon_{l}^{\prime}+i \epsilon_{l}^{\prime \prime}=n_{l}^{2}$ and arbitrary radii $a_{l}$ located in a uni- 
form medium with complex dielectric constant $\epsilon_{b}=\epsilon_{b}^{\prime}+i \epsilon_{b}^{\prime \prime}=n_{b}^{2}$ (Fig. 19), where $n_{l}=n_{l}^{\prime}+i n_{l}^{\prime \prime}$ and $n_{b}=n_{b}^{\prime}+i n_{b}^{\prime \prime}$ are the refractive indices of the cylinders and the background. The complex dielectric permittivities of the cylinders and the background can be arbitrary and may be frequency dependent.

In two dimensions, the solution of the electromagnetic field problem decouples into two fundamental polarizations, in each of which the field may be characterized by a single field component: $V(\mathbf{r})=E_{z}$ (for TM polarization) and $V(\mathbf{r})=H_{z}$ (for TE polarization). In the coordinate system that is used, the $z$ axis is aligned with the cylinder axes.

The field component $V$ satisfies the Helmholtz equation

$$
\nabla^{2} V(\mathbf{r})+k^{2} n^{2}(\mathbf{r}) V(\mathbf{r})=0 .
$$

For TM polarization, both $V(\mathbf{r})$ and its normal derivative $\boldsymbol{\nu} \cdot \nabla V(\mathbf{r})$ are continuous across all boundaries, while for TE polarization the corresponding boundary conditions are the continuity of $V(\mathbf{r})$ and its weighted normal derivative $\boldsymbol{\nu} \cdot \nabla V / n^{2}(\mathbf{r})$. Here, $n(\mathbf{r})$ denotes the refractive index of the relative medium and $\boldsymbol{\nu}$ is an unit outward normal vector.

In the vicinity of the $l$ th cylinder, we may represent the exterior field in the background medium (refractive index $n_{b}$ ) in local coordinates as $\mathbf{r}_{l}=\left(r_{l}, \theta_{l}\right)=\mathbf{r}-\mathbf{c}_{l}$, where $\mathbf{c}_{l}$ represents the center of the cylinder, and we write

$$
V(\mathbf{r})=\sum_{m=-\infty}^{\infty}\left[A_{m}^{l} J_{m}\left(k n_{b} r_{l}\right)+B_{m}^{l} H_{m}^{(1)}\left(k n_{b} r_{l}\right)\right] e^{i m \theta_{l}} .
$$

This local expansion is valid only in an annulus extending from the surface of the cylinder $l$ to the surface of the nearest adjacent cylinder.

\section{Figure 19}

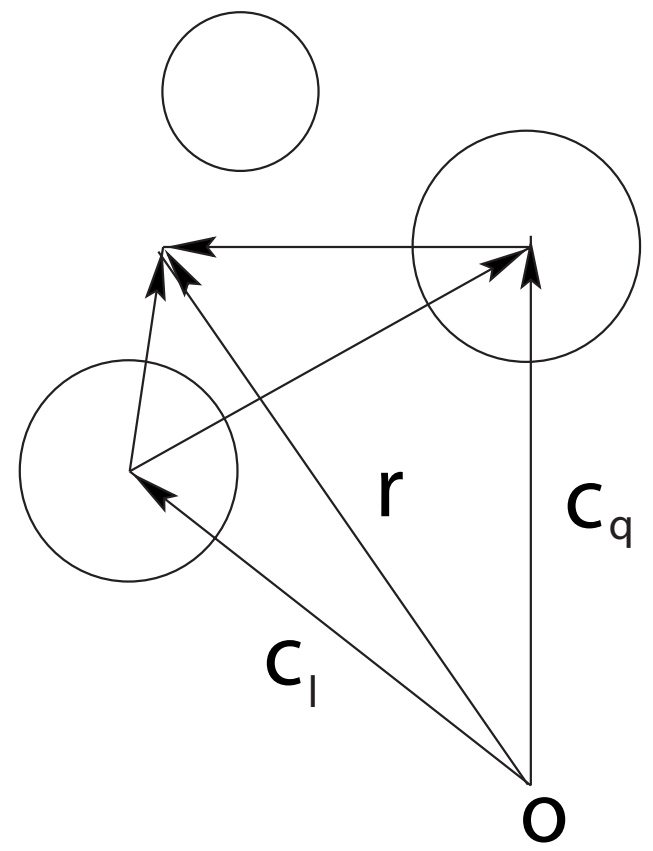

Geometry and local coordinate systems. 
The global field expansion (also referred to as a Wijngaard expansion), which is valid everywhere in the background matrix, comprises only outgoing cylindrical harmonic terms:

$$
V(\mathbf{r})=\sum_{q=1}^{N_{c}} \sum_{m=-\infty}^{\infty} B_{m}^{q} H_{m}^{(1)}\left(k\left|\mathbf{r}-\mathbf{c}_{q}\right|\right) e^{i m \arg \left(\mathbf{r}-\mathbf{c}_{q}\right)} .
$$

Correspondingly, the field inside any cylinder $l$ is written in an interior expansion:

$$
V(\mathbf{r})=\sum_{m=-\infty}^{\infty} C_{m}^{l} J_{m}\left(k n_{l}\left|\mathbf{r}-\mathbf{c}_{l}\right|\right) e^{i m \arg \left(\mathbf{r}-\mathbf{c}_{l}\right)} .
$$

Then, applying Graf's addition theorem [53] to the terms on the right-hand side of Eq. (A3) (see Fig. 19), we may express the global field expansion in terms of the local coordinate system for the $l$ th cylinder. Equating this with the local expansion (A2), we deduce the field identity (also known as the Rayleigh identity):

$$
A_{m}^{l}=\sum_{q=1, q \neq l}^{N_{c}} \sum_{p=-\infty}^{\infty} H_{m p}^{l q} B_{p}^{q}
$$

where

$$
H_{m p}^{l q}=H_{m-p}^{(1)}\left(k c_{l q}\right) e^{-i(m-p) \theta_{l q}}
$$

Here, $\left(c_{l q}, \theta_{l q}\right)$ are the polar coordinates of the vector $\mathbf{c}_{l q}=\mathbf{c}_{q}-\mathbf{c}_{l}$, the position of cylinder $q$ relative to cylinder $l$.

This is the first connection between the standing wave $\left(\left\{A_{m}^{l}\right\}\right)$ and outgoing $\left(\left\{B_{m}^{l}\right\}\right)$ multipole coefficients, one which follows solely from the system geometry. Equation (A5) indicates that the local field in the vicinity of cylinder $l$ is due to sources on all other cylinders $(q \neq l)$, the contributions of which to the multipole term of order $m-p$ at cylinder $l$ are given by $H_{m p}^{l q}$.

The second relation between the $\left\{A_{m}^{l}\right\}$ and $\left\{B_{m}^{l}\right\}$ multipole coefficients is obtained from the field continuity equations (i.e., the boundary conditions) at the interface of cylinder $l$ and the local exterior (A2) and interior field (A4) expansions. From these, we obtain

$$
\begin{aligned}
& B_{m}^{l}=R_{m}^{l} A_{m}^{l}, \\
& C_{m}^{l}=T_{m}^{l} A_{m}^{l},
\end{aligned}
$$

where the interface reflection and transmission coefficients, for both $E_{z}$ and $H_{z}$ polarization, are given by

$$
\begin{aligned}
& R_{m}^{l}=-\frac{\xi n_{l} J_{m}^{\prime}\left(k n_{l} a_{l}\right) J_{m}\left(k n_{b} a_{l}\right)-n_{b} J_{m}\left(k n_{l} a_{l}\right) J_{m}^{\prime}\left(k n_{b} a_{l}\right)}{\xi n_{l} J_{m}^{\prime}\left(k n_{l} a_{l}\right) H_{m}^{(1)}\left(k n_{b} a_{l}\right)-n_{b} J_{m}\left(k n_{l} a_{l}\right) H_{m}^{(1)^{\prime}}\left(k n_{b} a_{l}\right)}, \\
& T_{m}^{l}=-\frac{2 i /\left(\pi k a_{L}\right)}{\xi n_{l} J_{m}^{\prime}\left(n_{l} k a_{l}\right) H_{m}^{(1)}\left(k n_{b} a_{l}\right)-n_{b} J_{m}\left(k n_{l} a_{l}\right) H_{m}^{(1)^{\prime}}\left(k n_{b} a_{l}\right)},
\end{aligned}
$$

in which $\xi=1$ for TM polarization and $\xi=n_{b}^{2}(\mathbf{r}) / n_{l}^{2}(\mathbf{r})$ for TE polarization. 
To derive a simple closed form expression for the solution of the problem, we use partitioned matrix notation, introducing vectors $\mathbf{A}^{l}=\left[A_{m}^{l}\right]$ and $\mathbf{B}^{l}=\left[B_{m}^{l}\right]$ and expressing Eq. (A5) in the form

$$
\mathbf{A}^{l}=\sum_{q} \mathbf{H}^{l q} \mathbf{B}^{q},
$$

where $\mathbf{A}^{l}$ and $\mathbf{B}^{l}$ denote vectors of multipole coefficients for cylinder $l$. The matrix $\mathbf{H}$ is block partitioned according to $\mathbf{H}^{l q}=\left[H_{m p}^{l q}\right]$ for $l \neq q(\mathrm{~A} 6)$, and $\mathbf{H}^{l l}=[0]$, each block of which is a matrix of Toeplitz form. Correspondingly, the matrix forms of Eqs. (A7) and (A8) are

$$
\begin{aligned}
& \mathbf{B}=\mathbf{R A}, \\
& \mathbf{C}=\mathbf{T A},
\end{aligned}
$$

where $\mathbf{R}=\operatorname{diag} \mathbf{R}^{l}$ is a block diagonal matrix of diagonal matrices $\mathbf{R}^{l}=\operatorname{diag} R_{m}^{l}$, and with corresponding definitions applying for the transmission matrices.

Then, with the introduction of the partitioned vectors $\mathbf{A}=\left[\mathbf{A}^{l}\right], \mathbf{B}=\left[\mathbf{B}^{l}\right]$ and the partitioned matrix $\mathbf{H}=\left[\mathbf{H}^{l q}\right]$, we form the system of equations

$$
(\mathbf{I}-\mathbf{R H}) \mathbf{B}=0 .
$$

The problem has now been reduced to the solution of a generalized eigenvalue problem for matrix equation (A14). The nontrivial solutions of secular equation (A14) determine modes of the random system. Finding the nontrivial solutions of the linear system of equations (A14) requires that the determinant of the system matrix vanish:

$D=0, \quad$ where $D=\operatorname{det}\left(\mathbf{S}^{-1}\right)$

with

$\mathbf{S}^{-1}(\lambda)=(\mathbf{I}-\mathbf{R H})$.

Equivalently, this problem may be recast as a search for the poles of the scattering matrix $\mathbf{S}(\lambda)$ (i.e., solutions of $\operatorname{det} \mathbf{S}^{-1}(\lambda)=0$ ). Once the pole is located, the corresponding null vectors $\mathbf{B}$ of Eq. (A14) are the multipole coefficients of the scattered field, which are used to calculate the QB state profiles exterior to the scatterers by using Eq. (A3). The field inside a cylinder is calculated according to the interior expansions, Eqs. (A13) and (A4). The TLM poles must be searched in the $\left(\lambda, \epsilon_{c}\right)$ domain, given that the pump changes not only the imaginary part of the refractive index but the real part as well [Eq. (4)].

Formal system (A15) is of infinite dimension and so must be truncated to generate a computational solution, the accuracy of which is governed by the number of retained multipole coefficients $N_{m}=2 N_{\max }+1$, where $N_{\max }$ is the truncation order of the multipole series; i.e., only the terms corresponding to the cylindrical harmonics of order $n=-N_{\max }, \ldots, N_{\max }$ are retained.

\section{Appendix B: Finite Element Method}

We have also used the finite element method [60], implemented in a commercial software (Comsol), to solve wave equation (A1) and calculate the complex eigenvalues and eigenfunctions of the passive modes of the systems that were calculated by the multipole method. The method suitably applies for modeling pas- 


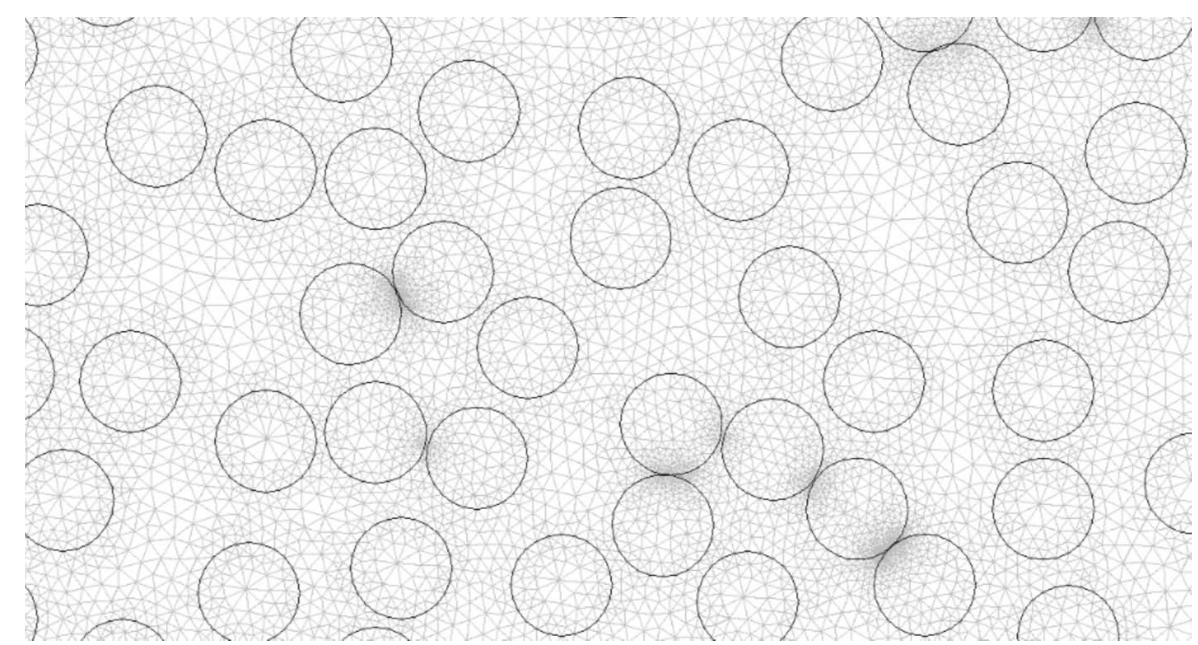

Close up of a typical mesh created by Comsol to describe the 2D random system of Fig. 2.

sive or active modes in a cavity, which is surrounded by perfectly matched layers [64] to simulate open boundaries. It is possible to obtain all the leaky modes, even the resonances characterized by a very small quality factor (as small as 5), in a reasonable computation time with a commercial PC, provided the size of the geometry is smaller than hundred times the wavelength. This is in contrast with the other methods described in this paper, which require much heavier computation.

One of the most important steps of the finite element method is the creation of the mesh that describes the system. Figure 20 shows a close up of a typical mesh calculated for the 2D random system of Fig. 2. The maximum size of elements must be smaller than seven times the wavelength [65].

\section{Acknowledgments}

P. Sebbah thanks the French National Research Agency which supports this work under grant ANR-08-BLAN-0302-01, the PACA region, and the CG06. A. A. Asatryan acknowledges the support from the Australian Research Council under its Centres of Excellence and Discovery Grants programs. A. D. Stone acknowledges support from the National Science Foundation under grant DMR0908437. H. E. Türeci acknowledges support from the Swiss NSF under grant PP00P2-123519/1.

\section{References}

1. D. S. Wiersma, “The smallest random laser," Nature 406, 132-135 (2000).

2. H. Cao, "Random lasers with coherent feedback," in Optical Properties of Nanostructured Random Media V. M. Shalaev, ed., Vol. 82 of Topics in Applied Physics (Springer-Verlag, 2002), pp. 303-330

3. H. Cao, "Lasing in random media," Waves Random Complex Media 13, 
R1-R39 (2003).

4. H. Cao, J. Y. Xu, Y. Ling, A. L. Burin, E. W. Seeling, X. Liu, and R. P. H. Chang, "Random lasers with coherent feedback," IEEE J. Sel. Top. Quantum Electron. 9, 111-118 (2003).

5. D. S. Wiersma, "The physics and applications of random lasers," Nat. Phys. 4, 359-367 (2008).

6. P. W. Anderson, "Absence of diffusion in certain random lattices," Phys. Rev. 109, 1492 (1958).

7. C. Gouedart, D. Husson, C. Sauteret, F. Auzel, and A. Migus, "Generation of spatially incoherent short pulses in laser pumped neodymium stoichiometric crystal powders," J. Opt. Soc. Am. B 10, 2358-2363 (1993).

8. N. M. Lawandy, R. M. Balachandra, A. S. L. Gomes, and E. Sauvain, "Laser action in strongly scattering media," Nature 368, 436-438 (1994).

9. W. L. Sha, C. H. Liu, and R. R. Alfano, "Spectral and temporal measurements of laser action of Rhodamine 640 dye in strongly scattering media," Opt. Lett. 19, 1922-1924 (1994).

10. H. Cao, Y. G. Zhao, H. C. Ong, S. T. Ho, J. Y. Dai, J. Y. Wu, and R. P. H. Chang, "Ultraviolet lasing in resonators formed by scattering in semiconductor polycristalline films," Appl. Phys. Lett. 73, 3656-3658 (1998).

11. H. Cao, Y. G. Zhao, S. T. Ho, E. W. Seelig, Q. H. Wang, and R. P. H. Chang, "Random laser action in semiconductor powder," Phys. Rev. Lett. 82, 22782281 (1999).

12. H. Cao, Y. G. Zhao, H. C. Ong, and R. P. H. Chang, "Far-field characteristics of random lasers," Phys. Rev. B 59, 15107-15111 (1999).

13. S. V. Frolov, Z. V. Vardeny, K. Yoshino, A. Zakhidov, and R. H. Baughman, "Stimulated emission in high-gain organic media," Phys. Rev. B 59, R5284-R5287 (1999).

14. H. Cao, J. Y. Xu, D. Z. Zhang, S.-H. Chang, S. T. Ho, E. W. Seelig, X. Liu, and R. P. H. Chang, "Spatial confinement of laser light in active random media," Phys. Rev. Lett. 84, 5584-5587 (2000).

15. H. Cao, Y. Ling, J. Y. Xu, C. Q. Cao, and C. Q. Cao, "Photon statistics of random lasers with resonant feedback," Phys. Rev. Lett. 86, 4524-4527 (2001).

16. A. Lagendijk, B. van Tiggelen, and D. S. Wiersma, "Fifty years of Anderson localization," Phys. Today 62(8), 24-29 (2009).

17. V. Milner and A. Z. Genack, "Photon localization laser: low-threshold lasing in a random amplifying layered medium via wave localization," Phys. Rev. Lett. 94, 073901 (2005).

18. K. L. van der Molen, R. W. Tjerkstra, A. P. Mosk, and A. Lagendijk, "Spatial extent of random laser modes," Phys. Rev. Lett. 98, 143901 (2007).

19. V. M. Apalkov, M. E. Raikh, and B. Shapiro, "Random resonators and prelocalized modes in disordered dielectric films," Phys. Rev. Lett. 89, 016802 (2002).

20. V. M. Apalkov M. E. Raikh, and B. Shapiro, "Almost localized photon modes in continuous and discrete models of disordered media," J. Opt. Soc. Am. B 21, 132-140 (2004).

21. S. Mujumdar, M. Ricci, R. Torre, and D. S. Wiersma, "Amplified extended modes in random lasers," Phys. Rev. Lett. 93, 053903 (2004).

22. S. Mujumdar, V. Türck, R. Torre, and D. S. Wiersma, "Chaotic behavior of a random laser with static disorder," Phys. Rev. A 76, 033807 (2007).

23. A. A. Chabanov, Z. Q. Zhang, and A. Z. Genack, "Breakdown of diffusion in dynamics of extended waves in mesoscopic media," Phys. Rev. Lett. 90, 
203903 (2003).

24. J. Fallert, R. J. B. Dietz, J. Sartor, D. Schneider, C. Klingshirn, and H. Kalt, "Co-existence of strongly and weakly localized random laser modes," Nat. Photonics 3, 279-282 (2009).

25. X. Jiang and C. M. Soukoulis, "Time dependent theory for random lasers," Phys. Rev. Lett. 85, 70-73 (2000).

26. C. Vanneste and P. Sebbah, "Selective excitation of localized modes in active random media," Phys. Rev. Lett. 87, 183903 (2001).

27. P. Sebbah and C. Vanneste, "Random laser in the localized regime," Phys. Rev. B 66, 144202 (2002).

28. C. Vanneste, P. Sebbah, and H. Cao, "Lasing with resonant feedback in weakly scattering random systems," Phys. Rev. Lett. 98, 143902 (2007).

29. C. Vanneste and P. Sebbah, "Complexity of two-dimensional quasimodes at the transition from weak scattering to Anderson localization," Phys. Rev. A 79, 041802(R) (2009).

30. X. Wu, W. Fang, A. Yamilov, A. A. Chabanov, A. A. Asatryan, L. C. Botten, and H. Cao, "Random lasing in weakly scattering systems," Phys. Rev. A 74, 053812 (2006).

31. H. E. Türeci, A. D. Stone, and B. Collier, "Self-consistent multimode lasing theory for complex or random lasing media," Phys. Rev. A 74, 043822 (2006).

32. H. E. Türeci, A. D. Stone, and L. Ge, "Theory of the spatial structure of nonlinear lasing modes," Phys. Rev. A 76, 013813 (2007).

33. H. E. Türeci, L. Ge, S. Rotter, and A. D. Stone, "Strong interactions in multimode random lasers," Science 320, 643 (2008).

34. L. Ge, R. Tandy, A. D. Stone, and H. E. Türeci, "Quantitative verification of ab initio self-consistent laser theory," Opt. Express 16, 16895 (2008).

35. H. E. Türeci, A. D. Stone, L. Ge, S. Rotter, and R. J. Tandy, “Ab initio selfconsistent laser theory and random lasers," Nonlinearity 22, C1-C18 (2009).

36. P. Sebbah, D. Sornette, and C. Vanneste, "Anomalous diffusion in twodimensional Anderson-localization dynamics," Phys. Rev. B 48, 1250612510 (1993).

37. F. A. Pinheiro, M. Rusek, A. Orlowski, and B. A. van Tiggelen, "Probing Anderson localization of light via decay rate statistic," Phys. Rev. E 69, 026605 (2004).

38. The idea of considering localized modes for random lasing can already be found in P. Sebbah, D. Sornette, and C. Vanneste, "Wave automaton for wave propagation in the time domain," Advances in Optical Imaging and Photon Migration, R. R. Alfano, ed., Vol. 21 of OSA Proceedings Series (Optical Society of America, 1994), p.68 (1994), and Ref. [39].

39. P. Sebbah, "A new approach for the study of wave propagation and localization," Ph.D. thesis (Université de Nice-Sophia Antipolis, 1993).

40. A. E. Siegman, Lasers (University Science Books, 1986).

41. A. Taflove, Computational Electrodynamics: the Finite-Difference TimeDomain Method (Artech House, 1995).

42. J. P. Berenger, "A perfectly matched layer for the absorption of electromagnetic waves," J. Comput. Phys. 114, 185-200 (1995).

43. X. Jiang and C. M. Soukoulis, "Localized random lasing modes and a path for observing localization," Phys. Rev. E 65, 025601(R) (2002).

44. A. G. Fox and T. Li, "Resonant modes in an optical maser," Proc. IRE 48, 1904-1905 (1960). 
45. A. G. Fox and T. Li, "Resonant modes in a maser interferometer," Bell Syst. Tech. J. 40, 453-488 (1961).

46. S. M. Dutra and G. Nienhuis, "Quantized mode of a leaky cavity," Phys. Rev. A 62, 063805 (2000).

47. J. C. J. Paasschens, T. Sh. Misirpashaev, and C. W. J. Beenakker, "Localization of light: dual symmetry between absorption and amplification," Phys. Rev. B 54, 11887-11890 (1996).

48. A. A. Asatryan, N. A. Nicorovici, L. C. Botten, M. C. de Sterke, P. A. Robinson, and R. C. McPhedran, "Electromagnetic localization in dispersive stratified media with random loss and gain," Phys. Rev. B 57, 13535-13549 (1998).

49. X. Jiang, Q. Li, and C. M. Soukoulis, "Symmetry between absorption and amplification in disordered media," Phys. Rev. B 59, R9007-R9010 (1999).

50. X. Wu, J. Andreasen, H. Cao, and A. Yamilov, "Effect of local pumping on random laser modes in one dimension," J. Opt. Soc. Am. B 24, A26-A33 (2007).

51. D. Felbacq, G. Tayeb, and D. Maystre, "Scattering by a random set of parallel cylinders," J. Opt. Soc. Am. A 11, 2526-2538 (1994).

52. E. Centeno and D. Felbacq, "Characterization of defect modes in finite bidimensional photonic crystals," J. Opt. Soc. Am. A 16, 2705-2712 (1999).

53. A. A. Asatryan, K. Busch, R. C. McPhedran, L. C. Botten, C. M. de Sterke, and N. A. Nicorovici, "Two-dimensional Green tensor and local density of states in finite-sized two-dimensional photonic crystals," Waves Random Complex Media 13, 9-25 (2003).

54. A. A. Asatryan, K. Busch, R. C. McPhedran, L. C. Botten, C. Martijn de Sterke, and N. A. Nicorovici, “Two-dimensional Green's function and local density of states in photonic crystals consisting of a finite number of cylinders of infinite length," Phys. Rev. E 63, 046612 (2001).

55. A. A. Asatryan, L. C. Botten, N. A. Nicorovici, R. C. McPhedran, and C. Martijn de Sterke, "Frequency shift of sources embedded in finite twodimensional photonic clusters," Waves Random Complex Media 16, 151165 (2006).

56. K. M. Lo, R. C. McPhedran, I. M. Bassett and G. W. Milton, "An electromagnetic theory of optical wave-guides with multiple embedded cylinders," J. Lightwave Technol. 12, 396-410 (1994).

57. T. P. White, B. Kuhlmey, R. C. McPhedran, D. Maystre, G. Renversez, C. Martijn de Sterke, and L. C. Botten, "Multipole method for microstructured optical fibers I: formulation,” J. Opt. Soc. Am. B 10, 2322-2330 (2002).

58. B. Kuhlmey, T. P. White, G. Renversez, D. Maystre, L. C. Botten, C. Martijn de Sterke, and R. C. McPhedran, "Multipole method for microstructured optical fibers II: implementation and results," J. Opt. Soc. Am. B 10, 23312340 (2002).

59. D. P. Fussell, R. C. McPhedran, C. Martijn de Sterke, and A. A. Asatryan, "Three-dimensional local density of states in a finite two-dimensional photonic crystal composed of cylinders," Phys. Rev. E 67, 045601(R) (2003).

60. J. Jin, The Finite Element Method in Electromagnetics (Wiley, 1993).

61. M. Sargent, M. O. Scully, and W. E. Lamb, Laser Physics (Addison-Wesley, 1982).

62. H. Haken, Light: Laser Dynamics (North-Holland, 1985), vol. 2.

63. Recently it was shown that one can define a variant of the CF states, termed the threshold constant flux (TCF) states, for which one TCF state is the TLM. Above threshold additional TCF states are needed to describe the las- 
ing modes, but in general fewer than for the CF states defined here. The above threshold theory using these TCF states is almost identical to the theory described in $[33,35]$. Details are in L. Ge, Y. Chong, and A. D. Stone, "Steady-state $a b$ initio laser theory: generalizations and analytic results," arXiv.org, arXiv:1008.0628v1 (submitted to Phys. Rev. A).

64. Ü. Pekel and R. Mittra, "A finite-element-method frequency-domain application of the perfectly matched layer (PML) concept," Microwave Opt. Technol. Lett. 9(3) 117-122 (2007).

65. P. Ambre, "Modélisation et caractérisation des fibres microstructurées air/ silice pour application aux télécommunications optiques," Ph.D. thèses (Université de Limoges, 2003).

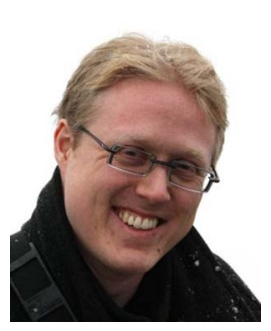

Jonathan Andreasen studied computational physics at Illinois State University and received his Ph.D. degree in physics and astronomy from Northwestern University, Evanston, Illinois, in 2009. He is a Research Affiliate in the Department of Applied Physics, Yale University, New Haven, Connecticut. Since 2010, he has been a Chateaubriand postdoctoral fellow in the Laboratoire de Physique de la Matière Condensée, Université de Nice-Sophia Antipolis, France. His research interests are computational electrodynamics, nonlinear dynamics and chaos, laser excitation, microcavity lasers, collective excitations, and applications of computer vision and genetic algorithms to optics.

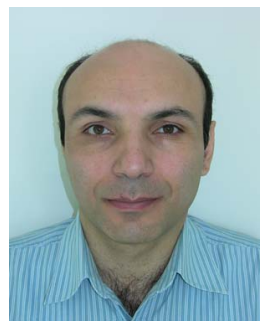

Ara Asatryan graduated with a master degree, first class honors from Yerevan State University in 1983, Armenia. His Ph.D. studies were done at the General Physics Institute of the Soviet Union Academy of Sciences, Moscow, supervised by Prof. Yu. A. Kravtsov. He got his Ph.D. in radiophysics including quantum electronics from the Radiophysics Institute of the Armenian Academy of Sciences in 1988. He started his employment as a lecturer at Yerevan State University in 1988 and become a reader in 1992. In 1994 he migrated to Australia and worked as a Research Associate at Macquarie University in 1995-1997 and at Sydney University in 1998-2001. Since 2002 he has worked at the University of Technology Sydney as a Research Associate and became a Senior Research Associate in 2004. His main area of research is in wave propagation in disordered media, Anderson localization, photonic crystals, and metamaterials. Some of his major results are the characterization of the effects of disorder in the propagation of waves in random photonic crystals, construction of the Green's function for finite photonic clusters and calculation of their local density of states, characterization of the effects of metamaterials on the Anderson localization, and calculation of the modes of random lasers. He is an Author of 70 refereed papers, four book chapters, and more than 100 conference presentations. He is the Senior member of the Optical Society of America and a member of the Australian Optical Society. He is a regular referee for Physical Review Letters, Physical Review A, B, and E, Optics Letters, Optics Express, and other major journals. 


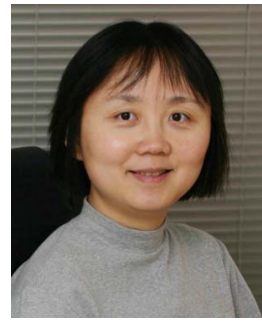

Hui Cao received the Ph.D. degree in applied physics from Stanford University, Stanford, California, in 1997. Currently, she is a Professor in the Department of Applied Physics and Physics, Yale University, New Haven, Connecticut. She has coauthored one book, three book chapters, three review articles and 130 journal papers. Dr. Cao received the David and Lucille Packard Fellow in 1999, the Alfred P. Sloan Fellow in 2000, the National Science Foundation (NSF) CAREER Award in 2001, the Friedrich Wilhelm Bessel Research Award of the Alexander von Humboldt Foundation in 2005, and the APS Maria Goeppert-Mayer Award in 2006. She is a fellow of the Optical Society of America (OSA) and the American Physical Society (APS).

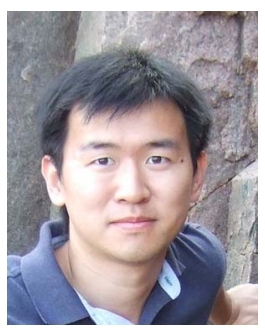

Li Ge received the B.S. degree in Physics from Peking University, China, in 2004. He then came to the United States and received his Ph.D. degree in Physics from Yale University in 2010. His doctoral research focused on self-consistent $a b$ initio laser theory (SALT) under the supervision of Prof. A. Douglas Stone. Currently he is working with Prof. Hakan Türeci as a postdoctoral associate in the Department of Electrical Engineering at

Princeton University.

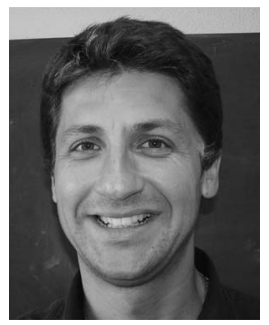

Laurent Labonté was born in Périgueux, France, on May 15, 1979. He received the Ph.D. degree in Electronics and Photonics from the University of Limoges (France), where he studied theoretically and experimentally the characteristics of the propagation in microstructure fiber. Since 2006, he has been a lecturer at the Laboratoire de Physique de la Matière Condensée, and his research interests have been in the design of original optic fibers. The applications of this research are in classical and quantum telecommunication. On the other hand, more generally, he is interested in studying the propagation of light waves in a guided structure.

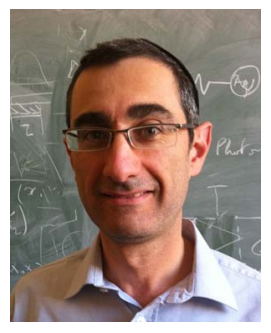

Patrick Sebbah is a researcher of the CNRS (Centre Nationale de la Recherche Scientifique) since 1994, presently at the Laboratoire de Physique de la Matiere Condensee, University of Nice-Sophia Antipolis, France. He received an education in general physics and laser physics. He graduated from the Ecole Nationale Superieure des Telecommunications de Paris (1988) and from the University Paris XI-Orsay (1989). He received his Ph.D. degree in physics from the University of Nice-Sophia Antipolis in 1993 for his dissertation on wave propagation and localization. He did his postdoctoral work at the Physics Department of Queens College, CUNY, New York, USA, in the group of Prof. A. Z. Genack. His research interests are light/acoustics wave/microwave propagation in complex media, multiple scattering and localization, random lasing, and nonlinear scattering. 
A. Douglas Stone is the Carl A. Morse Professor and Chair of Applied Physics and a Professor of Physics at Yale University. He joined the Yale faculty in 1986, was promoted to Professor in 1990, and previously served terms as Chair of Applied Physics and Director of the Division of Physical Sciences. Before coming to Yale he obtained a Master's degree in Physics and Philosophy from Oxford in 1978 (where he was a Rhode's Scholar), a Ph.D. in theoretical condensed matter physics from MIT in 1983, and did postdoctoral work at IBM's Yorktown Heights Laboratory (1983-1985). His Ph.D. work was in condensed matter theory, and the early part of his career focused on problems in mesoscopic electron systems and quantum fluctuations. His ground-breaking work with Patrick Lee on universal conductance fluctuations is one of the most cited works in all of physics from the 1980's ( 1900 ISI citations) and was recognized by the McMillan Award of the University of Illinois at Urbana for "outstanding contributions to condensed matter physics." For the past decade he has focused his research on optics, and specifically on microcavity optics and laser physics, and holds four patents in these areas. His group proposed the asymmetric resonant cavity laser in 1997, based on chaotic ray dynamics, and has developed since 2005 steady-state $a b$ initio laser theory (SALT). He was elected a Fellow of the Optical Society of America in 2010 for his work in laser physics and is also a Fellow of the American Physical Society and an honorary member of the Aspen Center for Physics.

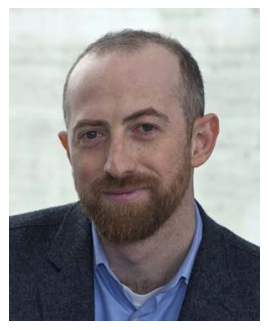

Hakan E. Türeci studied Physics at Bilkent University and received his Ph.D. from Yale University in 2003 for his dissertation on mesoscopic optics. He did his postdoctoral work in the Condensed Matter Theory group at Yale University and in the Institute for Quantum Electronics at ETH Zurich. In 2009, he was appointed as SNF Professor for Mesoscopic Quantum Optics at ETH. Since 2010, he has been an Assistant Professor in the Department of Electrical Engineering at Princeton University. His research interests span a wide range of theoretical problems in quantum optics and condensed matter physics.

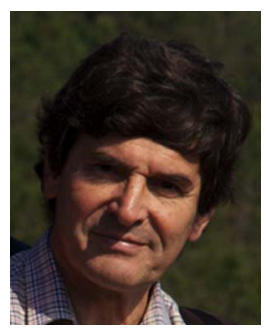

Christian Vanneste studied Physics at Orsay University and received his Ph.D. from Nice University in 1982 for his dissertation on the Josephson effect. After a postdoctoral stay at IBM Thomas J. Watson Research Center, he joined CNRS (Centre National de la Recherche Scientifique) in the Laboratoire de Physique de la Matière Condensée, University of Nice-Sophia Antipolis. His current research interests are wave propagation in complex media and random lasers. 